\title{
Meta-Representational Fluency: Math Majors' Visualization of the l'Hospital's Rule in a Dynamic Geometry Environment
}

\section{Gunhan Caglayan}

\section{(2) OpenEdition \\ Journals}

Electronic version

URL: http://journals.openedition.org/educationdidactique/2789

DOI: 10.4000/educationdidactique.2789

ISSN: $2111-4838$

Publisher

Presses universitaires de Rennes

\section{Printed version}

Date of publication: 6 December 2017

Number of pages: 63-101

ISBN: 978-2-7535-7318-5

ISSN: 1956-3485

\section{Electronic reference}

Gunhan Caglayan, « Meta-Representational Fluency: Math Majors' Visualization of the l'Hospital's Rule in a Dynamic Geometry Environment », Éducation et didactique [Online], 11-2 | 2017, Online since 06 December 2019, connection on 26 April 2019. URL : http://journals.openedition.org/ educationdidactique/2789; DOI : 10.4000/educationdidactique.2789 


\title{
META-REPRESENTATIONAL FLUENCY: MATH MAJORS' VISUALIZATION OF THE L'HOSPITAL'S RULE IN A DYNAMIC GEOMETRY ENVIRONMENT
}

\author{
Gunhan Caglayan \\ New Jersey City University, Mathematics Department \\ gcaglayan@njcu.edu
}

\begin{abstract}
The present qualitative study on mathematics majors' visualization of the l'Hospital's Rule in a dynamic geometry environment highlights student-generated representations in a theoretical framework drawn from visual thinking in calculus and meta-representational competences perspectives. Students developed their own personal meanings of the l'Hospital's formalism; their expression of meta-representational fluency occurred in different, yet not necessarily hierarchical modes of visualizations. Whereas global tangential visualization appeared in the form of static, successive, or simultaneous visualizations of the tangency points on both function graphs; functional visualization (local-tangential functional, static derivative functional, dynamic derivative functional) appeared as an additional key construct in students' treatment of the l'Hospital's Rule by primarily focusing on the function graphs, without taking tangent lines into account.
\end{abstract}

Keywords: undergraduate mathematics education, dynamic geometry software, visualization, limits and derivatives, l'Hospital's Rule. 


\section{INTRODUCTION}

In 1696, the French mathematician Guillaume François Marquis de l'Hospital published the differential calculus text Analyse des Infiniments Petits, a compilation of the brilliant Swiss physician and mathematician Johann Bernoulli's material, which also included a section on calculus of variations (Eves, 2006; Struik, 1963). "L'Hospital's Analyse is probably most famous as the source of l'Hospital's Rule-which should probably be renamed Bernoulli's rule-for calculating limits of quotients in the case where the limits of both numerator and denominator are zero" (Katz, 2009, p. 576). The origin of l'Hospital's Rule is based on the proposition: "Let $A M D$ be a curve $(A P=x, P M=y, A B=a)$ such that the value of the ordinate $y$ is expressed by a fraction, of which the numerator and denominator each become 0 when $x=a$, that is to say, when the point $P$ corresponds to the given point $B$. It is required to find what will then be the value of the ordinate BD" (p. 576) as illustrated in fig. la. In a modern text, the l'Hospital's Rule is defined as: "Suppose $f$ and $g$ are differentiable and $g^{\prime}(x) \neq 0$ on an open interval $I$ that contains $a$ (except possibly at $a$ ). Suppose that $\lim x \rightarrow a f(x)=0$ and $\lim x \rightarrow a g(x)=0$ or that $\lim x \rightarrow a f(x)= \pm \infty$ and $\lim x \rightarrow a g(x)= \pm \infty$ (In other words, we have an indeterminate form of type $\frac{0}{0}$ or $\left.\frac{\infty}{\infty}\right)$. Then $\lim _{x \rightarrow a} \frac{f(x)}{g(x)}=\lim _{x \rightarrow a} \frac{f^{\prime}(x)}{g^{\prime}(x)}$ if the limit on the right side exists (or is $\infty$ or $-\infty$ )" (Stewart, 2012, p. 302). A geometric illustration that accompanies the modern definition is based on the notion of slope of tangent lines (Fig. 1b).
Figure 1.

A geometric illustration of the l'Hospital's Rule

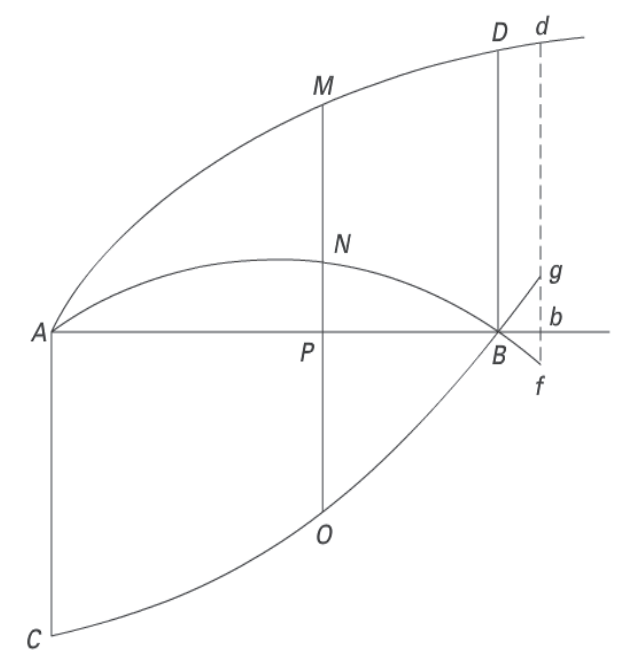

Fig. 1a. - Katz (2009, p. 577).
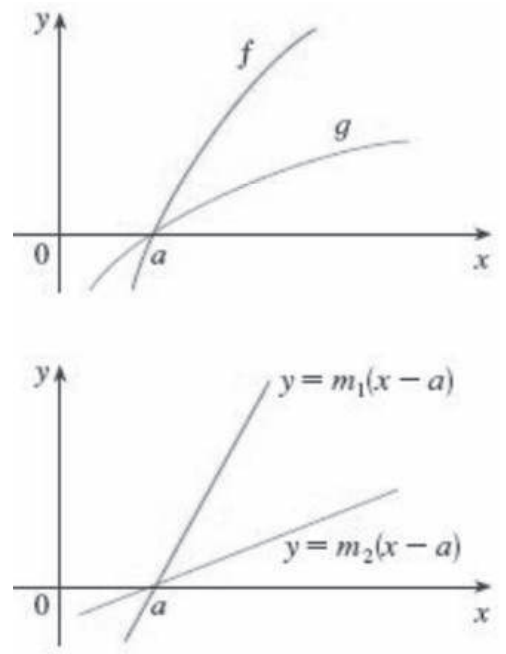

Fig. 1b. - Stewart (2012, p. 302).

\section{BACKGROUND AND RATIONALE FOR THE STUDY}

The focus of this study is on university students' visualization of the l'Hospital's Rule in a dynamic geometry software (DGS) and their reconciliation of the visualized, verbal, and written formalisms. In a case study on two calculus students' reinvention of the formal definition of limit, Swinyard (2011) postulated that "for one to determine a limit with 
certainty, he/she must employ algebraic techniques (e.g., rationalizing the numerator, factoring, applying L'Hospital's Rule) to the algebraic representation so as to eventually use direct substitution" (p. 100). Sofronas et al. (2011) reported L'Hospital's Rule as one of the topics omitted by the calculus experts from the curriculum, along with "related rates, surfaces of revolution, and logarithmic differentiation" (p. 135). Apart from these, the only articles referring to l'Hospital's Rule that appeared in mathematics education research journals are the ones that either report on historical perspectives (Kleiner, 2001; Struik, 1963) or the one that includes l'Hospital's Rule in a questionnaire as part of a largescale research study focusing in calculus in general (Bingolbali \& Monaghan, 2008).

Although derivative conceptions have been investigated by researchers in various contexts of derivatives, there are no studies reporting on university students' conceptions of derivatives in the context of l'Hospital's Rule. The purpose of the present report is to determine math majors' conceptions of the l'Hospital's Rule in a dynamic geometry environment. Due to the lack of empirical studies in mathematics education closely focusing on l'Hospital's Rule, the prior research informing the present study is portrayed under calculus students' conceptions of limits and derivatives.

\section{Limit Conceptions}

The present report is informed by research studies focusing on the teaching and the learning of the analysis concepts at the university level (GonzálezMartín et al. 2014; Schneider, 2001; Rogalski, 2015, 2016), in particular, the investigation of students' limit conceptions has been of a great interest for many researchers in the past (Davis \& Vinner, 1986; Gass, 1992; Harrington, 2006; Lecorre, 2015; Mamona-Downs, 2001; Robert, 1982; Robinet, 1983; Rogalski, 1990; Szydlik, 2000; Tall \& Vinner,
1981; Williams, 1991). Thinking about limit as an approximation via phrases like "as $x$ approaches $a$," "getting closer and closer to" is frequently adopted by calculus students (Davis \& Vinner, 1986; Williams, 1991). Przenioslo (2004) identified a number of limit conceptions held by university students, such as "neighbourhoods, graph approaching, values approaching, being defined at $x 0$, limit of $f$ at $x 0$ equals $f(x 0)$, and algorithms" (p. 103). According to Williams (1991), "conceptions of limit are often confounded by issues of whether a function can reach its limit, whether a limit is actually a bound, whether limits are dynamic processes or static objects, and whether limits are inherently tied to motion concepts" (p. 219). Cornu (1991) classified calculus students' conception of limit as static (based on intervals) and dynamic (based on movements). Yet the motion model of limit stands as a useful visual tool in sense-making of the limit of a function concept, it still may impede students' understanding of the static model of limit (Cornu, 1991). "Students often find a dynamic conception relatively easy and natural to develop, but progressing from this to a formal understanding of limits is much harder" (Thomas \& Holton, 2003, p. 365). In her study on calculus students' understandings and beliefs of the limit of a function, Szydlik (2000) classified students' limit conceptions as "intuitive static (The limit of a function is $L$ if whenever $x$ is close to the limiting value $s$, the function is close to $L$ ), motion (The limit of a function is $L$ if the function is getting closer and closer to $L$ as $x$ approaches s), and incoherent or inappropriate" (p. 268). Swinyard and Larsen (2012) conducted teaching experiments via which they proposed two theoretical constructs in an attempt to explain students' limit conception: "The $1^{\text {st }}$ construct relates to the need for students to move away from their tendency to attend first to the input variable of the function. The $2^{\text {nd }}$ construct relates to the need for students to overcome the practical impossibility of completing an infinite process" (p. 465). 


\section{Derivative Conceptions}

In modern texts, the derivative of a function $f$ at a number $a$ is defined as the limit of a quotient as (i) $f^{\prime}(a)=\lim _{h \rightarrow 0} \frac{f(a+h)-f(a)}{(a+h)-a}$ if the limit exists (Stewart, 2012, p. 146). Other representations of derivative include (ii) an instantaneous rate of change $\lim _{\Delta x \rightarrow 0} \frac{\Delta f}{\Delta x}=\lim _{x_{2} \rightarrow x_{1}} \frac{f_{2}-f_{1}}{x_{n}-x_{4}}$; (iii) a tangent line slope $m=\lim _{Q \rightarrow P} m_{P Q}=\lim _{Q \rightarrow P} \frac{y_{Q}-y_{P}}{x_{Q}-x_{P}} \quad$ (i.e., geometrically as the limit of the slopes of the secant lines); (iv) a function ; and (v) limited expansion of $1^{\text {st }}$ order $f(x)=f(a)+l(x-a)+o(x-a)$. Derivative as a tangent line slope can be interpreted geometrically as the limit of the slopes of the secant lines. NCTM (2000) emphasizes the importance of understanding "the conceptual foundations of limit, the area under a curve, the rate of change, and the slope of a tangent line, and their applications in other disciplines." The view of tangent line has been documented as to be closely associated in the context of circle; researchers reported that such an association might concatenate tangent line conception in the context of a curve (Biza, Christou, \& Zachariades, 2008; Biza \& Zachariades, 2010; Castela, 1995; Tall, 1987). "There are many students who can calculate derivatives of extremely messy functions but who cannot look at a graph and tell you where the derivative is positive and where it is negative" (Hughes-Hallett, 1991, p. 121). Drawing on a micro-ethnographic approach, Oehrtman (2009) identified five metaphor clusters revealing students' interpretations and conceptions of limits: (i) collapse metaphors (e.g., as the secant line becomes a tangent line in the limiting case, the two distinct points collapse to one same point); (ii) approximation metaphors (e.g., in the estimation of a function's limit); (iii) closeness metaphors (e.g., as the secant line becomes a tangent line in the limiting case, the amount of space between the two lines decreases); (iv) infinity as a number metaphors (e.g., scenarios involving growth and decay rate); (v) physical limitation metaphors.

Bingolbali, Monaghan and Roper (2007) reported that mathematics majors embraced the tangents conception of derivative while engineering majors favored for the rate of change (application) aspects. Several researchers reported that students tended to equate the derivative of a function to the equation of the tangent line to the same function graph at a particular point (Amit \& Vinner, 1990; Asiala, Cottrill, Dubinsky \& Schwingendorf, 1997). In a study with year 3 and 4 math majors, Mamolo and Zazkis (2012) found that "none of the participants was able to generalize for a square the derivative relationship evident in a circle. Further, when such a relationship was presented, only about half of the participants considered it as valid, and very few were able to extend the argument to a cube." (p. 176). In another study with 196 Year 12 students, Biza et al. (2008) identified three characterizations of tangency: (i) geometrical global perspective (students that are able to globally apply the geometrical properties of the tangent on the curve), (ii) analytical local perspective (students that possess a general view of tangency, without taking the geometric interpretation into account), and (iii) intermediate local perspective (transition between global and analytical). Artigue (1991) provided a set of a priori student conceptions of derivative rooted in that of a tangent line to a curve at a point $A$ :

(i) a line passing through $A$ but not crossing the curve in the neighbourhood of $A$,

(ii) a line having a double intersection with the curve at $A$,

(iii) a line passing through two points infinitely close to $A$ on the curve or the line which the curve becomes when one magnifies it in a neighbourhood of $A$,

(iv) the limit of the secants $(A M)$ as the point $M$ tends toward $A$ along the curve,

(v) the best linear approximation or the only linear approximation of the first order to the curve in a neighbourhood of $A$, 
Gunhan Caglayan

(vi) the line passing through $A$ whose slope is given by the derivative at $A$ of the function associated with the curve where the derivative is assumed to exist (pp. 174-175).

She further lists student conceptions regarding the derivative of the function $f$ at $x=a$ as the limit of the difference quotient, the slope of the tangent line at $x=a$, and the slope of "a highly magnified portion of the graph itself" (p. 175).

In an empirical study with 110 calculus student participants, Orton (1983) reported that most students were successful in executing the standard differentiation procedures or in finding the gradient of the tangent to a curve (e.g., $y=x^{3}-3 x^{2}+4$ ) at a value (e.g., at $x=3$ ). He reported that students had difficulties in the graphical interpretation of derivatives (e.g., the confusion between derivative evaluated at an $x$-value vs. the $y$-value of the point of tangency). He also identified major student difficulties and structural errors regarding the notions of average rate of change over an interval and the instantaneous rate of change. In a circle-secant situation, he observed that 43 out of 110 calculus students were unable to deduce that the secant line would ultimately become a tangent line in the limiting process (p. 237). He suggested, "in the normal approach to differentiations, students may need considerable help in understanding the tangent as the limit of the set of secants" (p. 237).

Ferrini-Mundy and Graham (1994) found that "students can perform the procedural tasks of calculus with rather astonishing success while displayed conceptual understandings are not what we would like to have in place" (p. 44). As an example, they provided an interview excerpt in which a student, Sandy, who correctly provided a "flawless description," yet unable to relate derivatives to tangent lines: "I'm not exactly sure... I can't remember exactly how it's related to the derivative... I remember doing it, but I can't remember exactly how" (p. 44). They suggested that "the availability of technology is a most promising factor in building curricula that could change this state of affairs. As the procedural tasks of calculus become less prominent in instruction, it would seem that whatever develops to take place of procedural emphasis should attend to the development of solid conceptual understanding of central ideas, understanding that allows students to solve problems in new domains" (p. 44).

\section{FOSTERING VISUAL THINKING IN CALCULUS: META-REPRESENTATIONAL COMPETENCE}

The guiding theoretical orientation is the multiple representations of mathematical concepts framework (Duval, 1993; Lesh, Post \& Behr; 1987) in the context of visual thinking in calculus (Zimmermann, 1991; Hughes-Hallet, 1991). As Hughes-Hallet (1991) put it, "students who are operating with few mental pictures are not really learning mathematics. Their calculus consists of a vast series of algorithms and complicated cataloging system which tells them which procedure to use when" (p. 121). She further stated that "the effort put into this kind of teaching is largely wasted: memorized algorithms are soon forgotten and, worse still, such courses perpetuate the idea that math involves doing calculations rather than thinking" (p. 121). Visualization, according to Tall (1991), can be used "to give a global gestalt for a mathematical concept, to show its strengths and weaknesses, its properties and non-properties, in a way that makes it a logical necessity to formulate the theory clearly" (p. 18). Zimmermann (1991) offered a list of prerequisites for visual thinking in calculus:

- understand algebra and geometry as alternative languages for the expression of mathematical ideas;

- understand the rules and conventions associated with mathematical graphics;

- extract specific information from diagrams;

- represent and interpret data graphically;

- plot functions intuitively and with the aid of a computer (p. 127-128). 
He goes on to state, "conceptually, the role of visual thinking is so fundamental to the understanding of calculus that it is difficult to imagine a successful calculus course which does not emphasize the visual elements of the subject" (p. 136). In a similar line of thinking, Bremigan (2005) stated, "calculus teachers often assume that their students have these prerequisite skills and that students appreciate the important role of reasoning with visual representations" (p. 249). Zimmerman (1991) further postulated that successful solutions to calculus problems are the ones that are accompanied by meaningful visual representations and graphs: "Of all undergraduate mathematics courses, none offers more interesting and varied opportunities for visualization than calculus... With computers and graphing calculators widely available, it is possible to use visualization in ways that would not be practical otherwise" (pp. 127, 136).

Aligned with the theories on visualizations in calculus presented above, the present report also draws from diSessa's (1988, 2004) theoretical perspective on meta-representational competences (MRC). diSessa (2004) asserted that "metarepresentation may be an important component of deeper understanding of any representation" (p. 299) and that "MRC makes the learning of representations seem sensible to students, hence it may contribute to motivation" (p. 327). "Computers provide an excellent medium for designing activities that build and integrate pieces of knowledge" (diSessa, 1988, p. 51). Students possessing metarepresentational competence (MRC) can:

- "MRC1. Invent or design new representations.

- MRC2. Critique and compare the adequacy of representations and judge their suitability for various tasks.

- MRC3. Understand the purposes of representations generally and in particular contexts and understand how representations do the work they do for us.

- MRC4. Explain representations (i.e., the ability to articulate their competence with the preceding items).
- MRC5. Learn new representations quickly and with minimal instruction" (diSessa, 2004, p. 293).

diSessa (2004) further argued that use of technology and new computer-based visualization techniques in the classroom activities would be prone to the further involvement of MRC as well (p. 297).

\section{CONTEXT AND METHODOLOGY}

The present study reports on university math majors' explorations of selected tasks on l'Hospital's Rule using GeoGebra, a free mathematics software that intertwines geometry, algebra, and spreadsheets environments. The rationale for using a DGS for the exploration of the aforementioned domain of calculus is inspired from the view of experimental mathematics (Borwein \& Bailey, 2003; Borwein, 2005; Sinclair, 2008). In this approach, the computer technology is used for:

- "Gaining insight and intuition;

- Discovering new patterns and relationships;

- Graphing to expose math principles;

- Testing and especially falsifying conjectures;

- Exploring a possible result to see if it merits formal proof;

- Suggesting approaches for formal proof;

- Computing replacing lengthy hand derivations;

- Confirming analytically derived results." (Borwein, 2005, p. 76.)

Qualitative-descriptive interview data (Patton, 2002) were collected over three years in a university in the United States, as part of a research project titled Geometry-Calculus-Linear Algebra Connections in a Dynamic Environment. The data for the analysis of the l'Hospital's Rule came from the videotapes of eighteen sets of two-hour interview sessions in a computer lab that included eight mathematics majors who completed the calculus sequence, who were interviewed by the author individually on separate days. Students were familiar with the software as they had already used it during the former set of interview 
Gunhan Caglayan

sessions on limits of functions; they were not provided any training or instructional help during the interviews. During the interviews, the students were asked to use GeoGebra to:

- solve the given problems both algebraically and with GeoGebra;

- indicate their problem solving procedure on GeoGebra;

- explain their reasoning with reference to their work on GeoGebra,

in agreement with the research objective. Aligned with the procedures described by Ferrini-Mundy and Graham (1994), "students were asked to complete the tasks and to think aloud as they did so" in order to "help the interviewer understand the student's thought process" (p. 33). Each interview session was videotaped as the participants worked on the mathematics problems algebraically and performed the graphs and constructions on the GeoGebra software. All names of participants are pseudonyms.

The qualitative interviews were based on a semi-structured interview model (Bernard, 1994; Kvale, 2007) in the course of which the interviewer "interacted with probes and questions" (FerriniMundy \& Graham, 1994) on the interviewees' responses. During each interview session, the interviewer did not intervene at all, nor corrected mathematical errors or proposed instructional help. "Methodologies employed in studies of this type are often qualitative and descriptive, based on interviews with students as they complete mathematical tasks... The intention is to provide rich and defensible descriptions of student understandings that can serve as springboards for acknowledging the great complexities to be understood in learning about student knowledge" (Ferrini-Mundy \& Graham, 1994, p. 32). All of the interviews were transcribed and a preliminary thematic analysis (Boyatzis, 1998) was carried out after each interview session by generating a written summary of the session with time stamps (Izsak, Caglayan \& Olive, 2009) in order to find possible themes for a more detailed analysis. These written summaries also contained comments about any significant events that are relevant to the research study, and screen shots from the video when needed for clarification or highlight throughout the subsequent data analyses (ibid.).

Upon the completion of data transcription, the corpus of interview data (transcribed interview data, research participants' algebraic solutions along with their work on GeoGebra) was reviewed multiple times, in accordance with constant comparative methodology (Glaser \& Strauss, 1967). The rationale for using constant comparison methodology is based on Glaser \& Strauss' proposition: "Control over similarities and differences is vital for discovering categories, and for developing and relating their theoretical properties, all necessary for the further development of an emergent theory" (1967, p. 55). Data analysis primarily focused on math majors' approaches in visualizing the mathematical ideas in GeoGebra platform in an attempt to identify students' sense-making and understanding of the l'Hospital's Rule in a dynamic geometry environment, in accordance with the research objective.

\section{RESULTS: COMPUTING LIMITS USING L'HOSPITAL'S RULE}

This chapter presents the findings on math majors' understanding of the l'Hospital's Rule in a dynamic geometry environment. Overall, math majors provided a diversity of approaches in their solutions for the limit problems of various indeterminate forms requiring the use of l'Hospital's Rule: (a) Forms that are equivalent to the $0 / 0$ indeterminate form: $\operatorname{im}_{x \rightarrow 0} \frac{1}{x} \sin \pi x, \lim _{x \rightarrow 0} \frac{5^{x}-4^{x}}{3^{x}-2^{x}}$ and $\lim _{x \rightarrow 0} \frac{8^{x}-2^{x}}{6^{x}-3^{x}}$; (b) The $0 \cdot \infty$ indeterminate form: $\lim _{x \rightarrow 1^{+}} \ln x \tan \frac{\pi x}{2}$ and $\lim _{x \rightarrow \infty} x \sin \frac{1}{x}$; (c) The $\infty-\infty$ indeterminate form: $\lim _{x \rightarrow 1^{+}}\left(\frac{x}{x-1}-\frac{1}{\ln x}\right)$ and $\lim _{x \rightarrow 0^{+}}\left(\frac{1}{x}-\frac{1}{e^{x}-1}\right)$. 


\section{A-Priori Analysis: Tangential vs. Local- Tangential Visualizations}

In accordance with the theoretical framework (visual thinking in Calculus and MRC), a computer-based exploration of the l'Hospital rule was fundamental, mainly to gain insight into students' thinking and ways of understanding of this very important theorem of Calculus. This section outlines the theoretical constructs that demonstrate the different ways that the l'Hospital's Rule could be visualized in a dynamic environment. In explaining these constructs, all snapshots offered in this section are based on the limit problem $\lim _{x \rightarrow 1} \frac{f(x)}{g(x)}$ calling for the use of l'Hospital's Rule where $f(x)=\arctan (\ln x)$ and $g(x)=x^{3}-x$ denote the numerator function and the denominator function, respectively ${ }^{1}$.

\section{Global Tangential Visualization}

This type of visualization is based on the construction of the tangent lines on the numerator and the denominator functions, and forming the ratio of the slopes of the tangent lines. Within global tangential visualization, there are three possibilities: (a) Static tangential visualization: Upon graphing the numerator function and the denominator function, the student identifies the common tangency point which is then used to graph the tangent lines for both curves, as depicted in Snapshot 1. The answer to the limit problem is then retrieved as the ratio of the slopes of the two tangent lines as 1/2. (b) Successive (consequential) tangential visualization (PseudoDynamic): Unlike the static tangential visualization in which a common (single) point of tangency is used, in this type of visualization, the student uses two separate tangency points (Snapshot 2a), which are then dragged on each curve in succession, until the two tangency points meet at the common point of tangency (Snapshot 2b). (c) Simultaneous (coherent) tangential visualization (Dynamic): Similar to the successive tangential visualization, the student uses two separate tangency points, however, in this case, one of the tangency points is plotted in such a way that it depends on the other tangency point. The student first plots tangency point A on curve $f$, followed by the construction of tangency point $\mathrm{B}$ on curve $g$ by typing $\mathrm{B}=(x(\mathrm{~A}), \mathrm{g}(x(\mathrm{~A}))$ as depicted in Snapshot 3a (Alternatively, point B could also be constructed as the intersection of the vertical line $x=x(A)$ with curve $g)$. Because both points of tangency have the same abscissa, dragging Point A will also cause Point B to move until both points meet at the common tangency point $(1,0)$ as before (Snapshot 3b).

\section{Functional Visualization}

This type of visualization is based on the construction of the numerator and the denominator functions without referring to tangent lines. Within functional visualization, there are three possibilities: (a) LocalTangential (Functional) Visualization: In this approach, the primary focus of consideration is the numerator and the denominator function graphs (of the original function), without taking into account the tangent lines. A student embracing this approach would continually zoom-into the coordinate plane until both curves look locally-linear, enabling the student to assign a slope value for both the numerator and the denominator functions. As demonstrated in Snapshot 4, the locally-linear looking curves $f$ and $g$ have respective slopes of 1 and 2, which can easily be determined by counting the nice squares that form. The student then determines the answer to the original problem by forming the ratio of these slopes, namely as 1/2. (b) Derivative Functional Visualization (Static): The sought value is determined by graphing the ratio of the derivative of the numerator function and the derivative of the denominator function. To 
highlight the answer, the student might take the additional step of constructing a point on the graph of the curve $\frac{f^{\prime}}{g^{\prime}}$ at $x=1$, as depicted in Snapshot 5 . (c) Derivative Functional Visualization (Dynamic): Unlike the static derivative functional visualization, the student separately graphs the curves $f^{\prime}$ and $g^{\prime}$; followed by the plotting of Point A on curve $f^{\prime}$ and Point B on curve $g^{\prime}$ (which depends on the abscissa of Point $\mathrm{A})$ by typing $\mathrm{B}=\left(x(\mathrm{~A}), \mathrm{g}^{\prime}(x(\mathrm{~A}))\right.$ as depicted in Snapshot $6 \mathrm{a}$ (It is also possible to construct Point B via the intersection of the vertical line $x=x(A)$ with curve $\left.g^{\prime}\right)$. As is the case with dynamic simultaneous tangential visualization, dragging Point A will also cause Point $\mathrm{B}$ to move until both points have the desired abscissa value of $x=1$ (Snapshot 6b). To highlight the answer, the student might take the extra step of calculating the ratio of the ordinates by typing $y(\mathrm{~A}) / y(\mathrm{~B})$ as depicted in Snapshot 6c. Metarepresentational fluency is defined as a student's ability to think deeply about and to connect many representations of derivatives such as instantaneous rate of change, slope of tangent line, derivative as a function, etc., in particular, in the context of l'Hospital's Rule; and to make sense within and among these representations.

\section{Forms that are equivalent to the $\frac{0}{0}$ indeterminate form}

In their explorations of the forms that are equivalent to the $0 / 0$ indeterminate form (e.g., $\lim _{x \rightarrow 0} \frac{1}{x} \sin \pi x$ ), most students immediately recognized the $\infty \cdot 0$ uncertainty and applied the L'Hospital's Rule upon converting it to the $\frac{0}{0}$ form. Anna, for instance, explained that she saw this to be of the $\frac{0}{0}$ form by first algebraically writing her expression as $\frac{\sin \pi x}{x}$. Prior to evaluating the limit algebraically, she used the $\operatorname{syntax}^{2} \operatorname{Limit}\left[(1 / x) * \sin \left(\pi^{*} x\right), 0\right]$, which was something she had discovered earlier in another limit problem, and obtained the value of the limit (Fig. 2a). Anna produced the graph of $f(x)=\frac{\sin \pi x}{x}$ (Fig. 2b) and constantly zoomed in to inspect the value of the limit (Fig. 2c): "As $x$ goes to zero... zooming in to see where the $y$-value is going [Fig. 2d] so I am gonna go with pi." Upon the interviewer's question "so you think it's pi?" she responded "that's an assumption yeah but I will tell you when I do the math... it's pi [(Fig. Al $\left.)^{3}\right]$."

Figure 2.

Anna's demonstrations

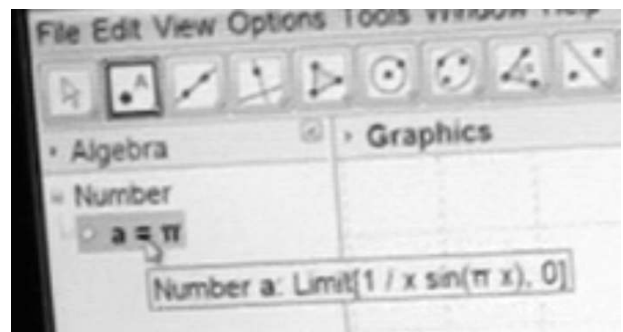

Fig. $2 a$.

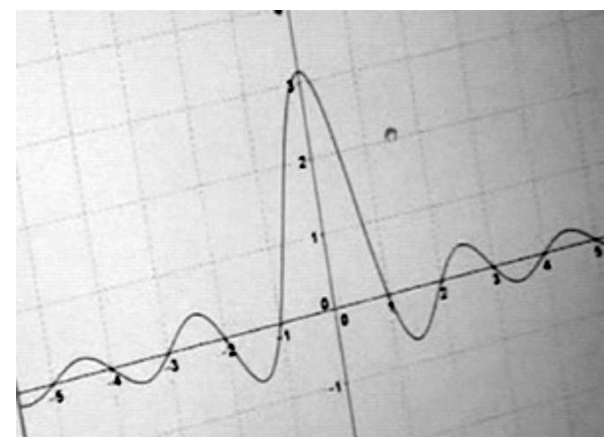

Fig. $2 b$.

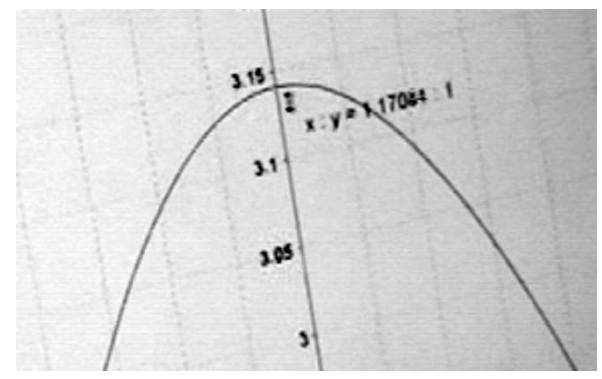

Fig 2c. 


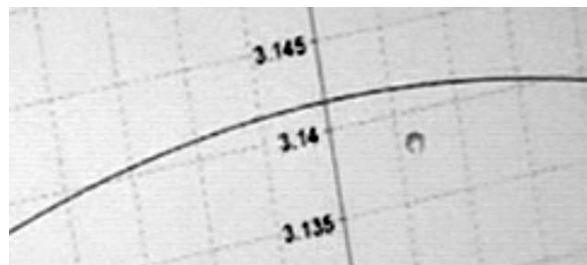

Fig. 2d.

While there was no connection to the l'Hospital's Rule in Anna's graphical demonstrations, Andy came up with an illustration of the l'Hospital's Rule in a limit expression of the indeterminate form. He first graphed the expression by typing the syntax $\left(5^{\wedge} x-4^{\wedge} x\right) /\left(3^{\wedge} x-2^{\wedge} x\right)$ which produced function $f$. He explained how he would visualize the l'Hospital's Rule in the investigation of $\lim _{x \rightarrow 0} \frac{5^{x}-4^{x}}{3^{x}-2^{x}}$ as:

"As $x$ goes to zero it looks like the $y$-value is about... $0.5 \ldots$ Using the l'Hospital's Rule I will get the derivative of the top and the derivative of the bottom [types the syntax derivative $\left[5^{\wedge} x-4^{\wedge} x\right]$ and derivative $\left[3^{\wedge} x-2^{\wedge} x\right]$ respectively and obtains the graphs of $g$ and $h] \ldots$ because l'Hospital's Rule says that if I take the derivative of the top and the derivative of the bottom when we have the zero over zero limit... and plug in zero afterwards... after I do that... It should give you the same answer... I wanna graph the derivative of the top divided by the derivative of the bottom and see that as $x$ goes to zero it should be the same as the original graph [types the syntax $\mathrm{g} / \mathrm{h}$ and obtains the graph of function p (Fig. 3a)]. I am gonna hide the $g$ of $x$ and the $h$ of $x$. As we can see the derivative [pointing to $\mathrm{g}$ ] over the derivative [pointing to $\mathrm{h}$ ] is this function [highlighting the graph of p (Fig. 3b)] and we can see as $x$ approaches zero the limit of that derivative over derivative should be the same as the original function as $x$ approaches zero. It should be the same limit. That derivative over derivative [highlighting p] intersects the same point as the original function intersects at [highlighting both $\mathrm{f}$ and $\mathrm{p}$ ]."
Figure 3.

Andy's work

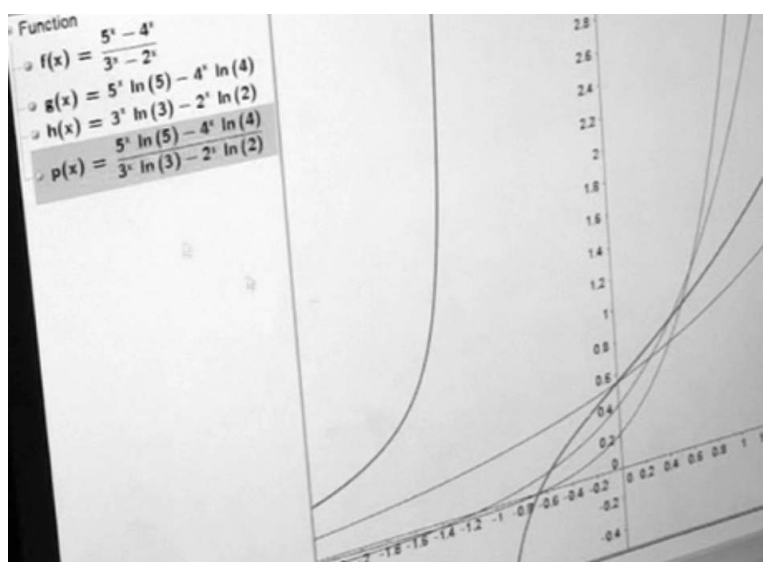

Fig. 3a.

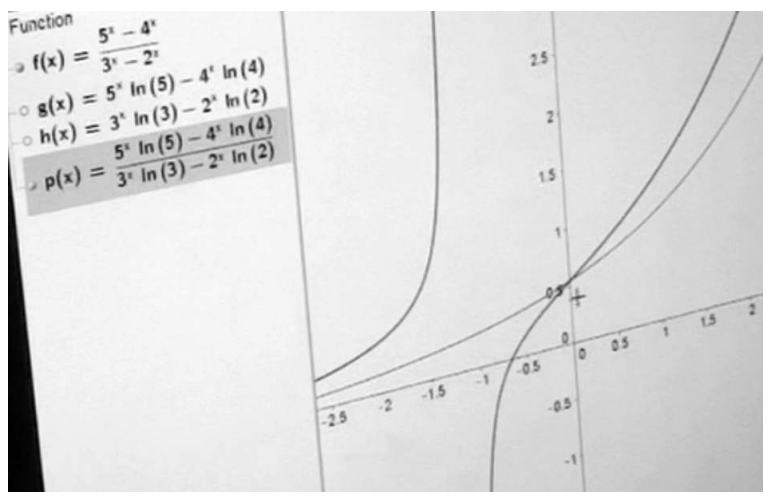

Fig. $3 b$

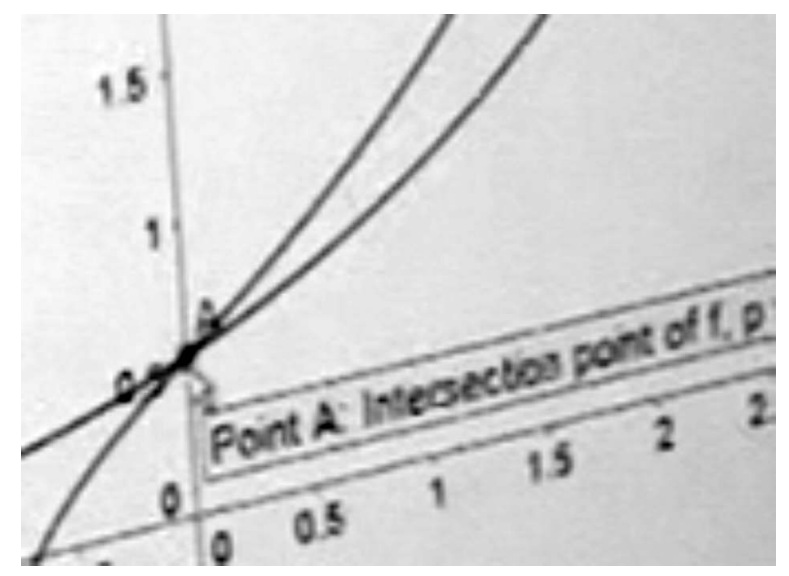

Fig. $3 c$ 
When the interviewer asked what the limit was, Andy responded: "The limit will be the intersection of these graphs [plots the intersection point using the Intersect Two Objects Tool (Fig. 3c)] so the limit would be point five five zero three four [pointing to the $y$-coordinate of point A]". Upon the interviewer's probing "What do you think about the limit... is it a rational or irrational number," Andy responded: "I would say irrational... I just have to set the limit equal to zero [pointing to the expression for function $p$ ] and then I'll just plug in zero for all these [respectively pointing to $5 \mathrm{x}, 4 \mathrm{x}, 2 \mathrm{x}, 3 \mathrm{x}$ ] and I'll get [works it out algebraically (Fig. A2)] so this would be my exact solution."

Remark that although the function $f$ is not defined at $x=0$, Andy was still able to plot the intersection of $f$ and $p$ at $x=0$ as point A (Fig. 3c). In a similar problem $\lim _{x \rightarrow 0} \frac{8^{x}-2^{x}}{6^{x}-3^{x}}$ of the form $\frac{0}{0}$, when the interviewer asked Glen to come up with a graphical illustration of the l'Hospital's Rule, Glen's first instinct was to graph the top function and the bottom function separately, by typing the syntaxes $\left(8^{\wedge} x-2^{\wedge} x\right)$ and $\left(6^{\wedge} x-3^{\wedge} x\right)$, respectively. He obtained the graphs of the induced ${ }^{4}$ functions $f$ and $g$; he constantly zoomed into the graph (Fig. 4a) and then suggested to plot points on the function graphs and use those points to construct tangent lines, which he did using the Tangents Tool in the Lines category. Upon the interviewer's question how he thought about plotting tangent lines, he stated that it was similar to a previous activity in which he explored tangent line as an approximation of secant lines. He further stated that the graph looked almost like a straight line when he repeatedly zoomed into the point of interest, indicative of his awareness of "local linearity" (Dick \& Edwards, 2008, p. 268). "Repeated magnifications of a differentiable function's graph centered at a particular point reveal quite strikingly this characteristic property of differentiability without the extra cognitive burden of dealing with the additional notion of a tangent line" (p. 268). Yet the interviewer did not probe on whether the induced function was differentiable at $x=0$ or not, this still did not prevent Glen from constructing a tangent line. Glen then moved the draggable points of tangency $A$ and $B$ in a consequential manner towards the origin, i.e., the point of interest; zoomed further in until the graphs of $f$ and $g$ looked even more locally linear, and used the Slope Tool to measure the slopes of the tangent lines (Fig. 4b). Finally, upon typing the syntax $1.38629 / 0.69315$, which produced $\mathrm{c}=1.99999$, he concluded that the limit should be 2 .

Figure 4.

Glen's graphs of the induced functions and the tangent lines

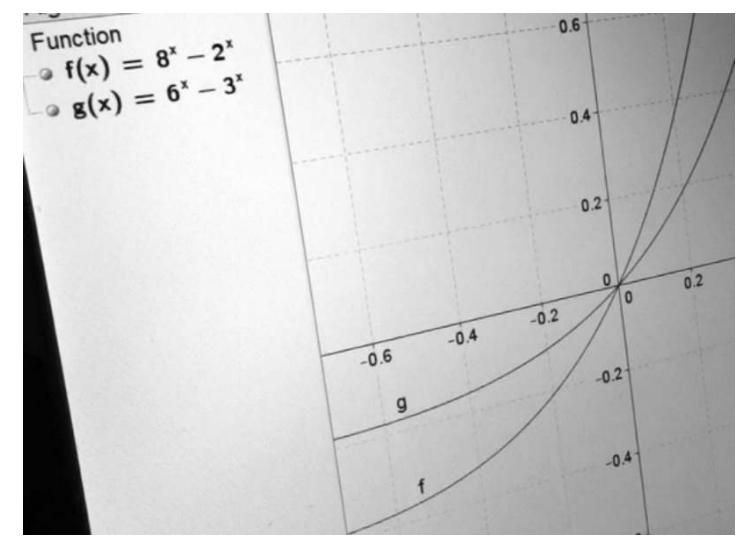

Fig. $4 a$.

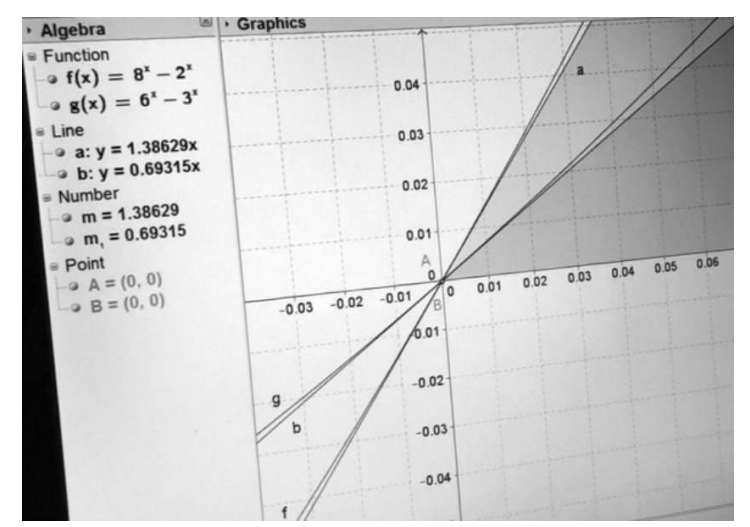

Fig. $4 b$. 
The analysis indicates that Andy's conception of derivative in the context of visualizing the l'Hospital's Rule (of original form $0 / 0$ ) was the "ratio of the derivatives of the functions" (Dick \& Edwards, 2008, p. 269) whereas Glen's conception of derivative in the same context was founded in the "ratio of slopes of the [tangent] lines" (p. 269). In light of the theoretical constructs proposed in the present report, Glen's visualization could be categorized as of successive (consequential) tangential type and Andy's visualization as derivative functional (static) type as his primary focus of consideration was the function graphs, without taking into account the tangent lines.

Gilda, Macy, Lindsey, Kyra, and Sharon developed a variety of visualizations and personal meanings for the $\lim _{x \rightarrow 0} \frac{8^{x}-2^{x}}{6^{x}-3^{x}}$ problem of $0 / 0$ indeterminate form. Gilda, for instance, graphed the induced function ${ }^{5}$ by typing $\left(8^{\wedge} x-2^{\wedge} x\right) /\left(6^{\wedge} x-3^{\wedge} x\right)$ and used the Intersect Two Objects Tool to plot the $y$-intercept, which appeared as "A undefined" on the algebra sidebar (Fig. 5a). This stimulated Gilda's willingness to solve the problem algebraically (Fig. A3). Although a graphical illustration of the l'Hospital's Rule was not demonstrated, Gilda still was able to confirm her algebraic result by stating "it looks like it's 2 on the graph as well... when I zoomed in like this [pointing to the $y$-intercept of the function graph (Fig. 5a)]." Macy followed almost the same strategy, except in her case she defined Point A by typing the syntax $(0, f(0))$ instead of using the Intersect Two Objects Tool, which still appeared as "A undefined" on the algebra sidebar (Fig. 5b). Lindsey preferred to start with the algebraic approach by which she obtained 2 as her answer to the limit problem (Fig. A4). She then used the software as a way of checking her answer by graphing the induced function (Fig. 5c) without plotting any point, while commenting "it looks like it is 2 [pointing to the $y$-intercept of the function graph]."
Figure 5.

Gilda (a), Macy (b), and Lindsey's (c) work

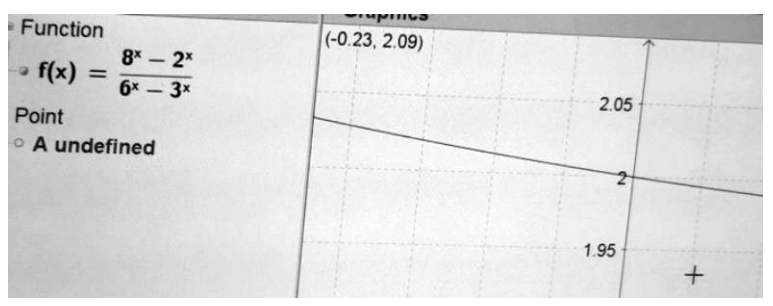

Fig. 5a.

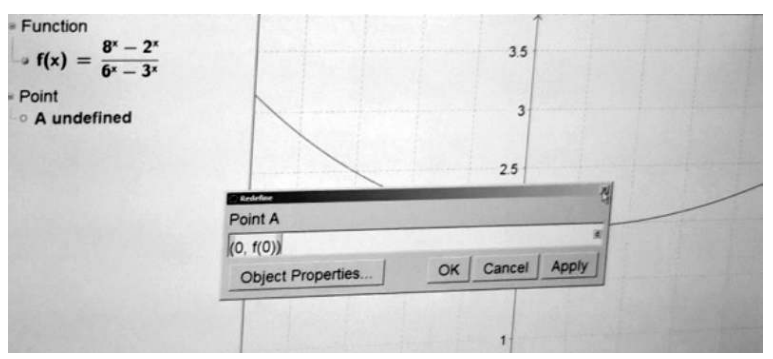

Fig. $5 b$.

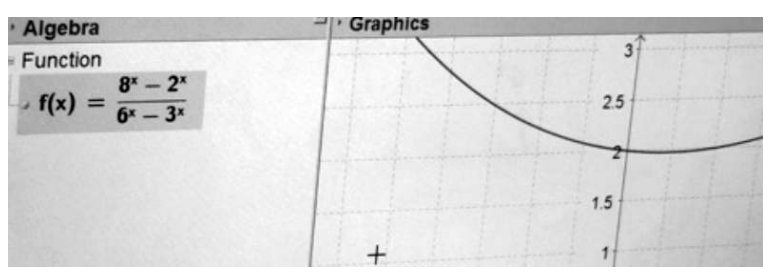

Fig. 5c.

Kyra graphed the induced function and obtained "b undefined" and "A undefined," respectively, when she entered the syntax Limit[f,0] and the $y$-intercept via the Intersect Two Objects Tool, respectively (Fig. 6a). When the interviewer probed on Kyra's preference to start with the graphical approach, she responded, "I wanna show that the limit is equal to $2 \ldots$ applying the l'Hospital's Rule would make it more complicated though wouldn't it?" She then suggested graphing the numerator and the denominator function graphs separately, in an attempt to visualize the l'Hospital's Rule, in coordination with her algebraic work. She explained, "as $x$ goes to zero they both go to zero so I am gonna find the derivatives by making the tangent lines [zooms into the graph and types the syntaxes Tangent $[(0,0), \mathrm{g}]$ and Tangent $[(0,0), \mathrm{h}]$ 
respectively (Fig. 6b-c)]." Kyra then commented, "doing the tangent lines shows that it's continuous at that point... I will also calculate the slopes [selects the Slope Tool and finds the slope of each tangent line (Fig. 6d)]." Upon the interviewer's probing why she measured the slopes of the tangent lines, she responded, "so I am just gonna type them as a fraction [types $\mathrm{m} / \mathrm{n}$ and $\mathrm{d}=2$ appears on the algebra sidebar] so the ratio of the slopes will give you 2 which is the limit of the original function."

Figure 6.

Kyra's static tangential visualization

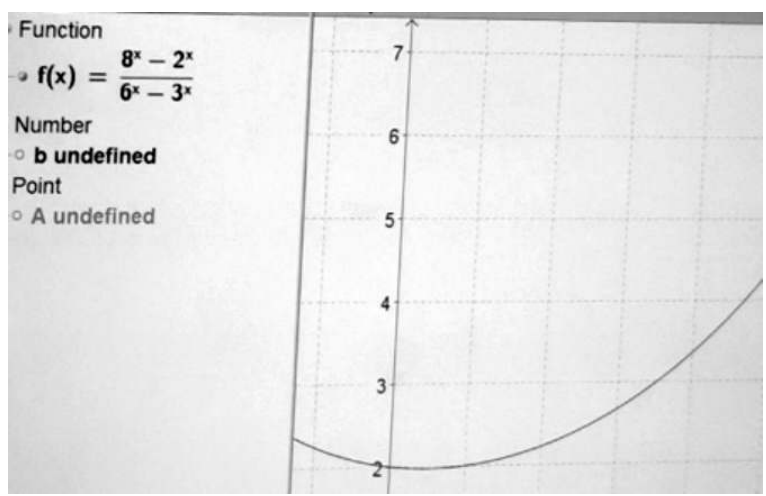

Fig. 6a.

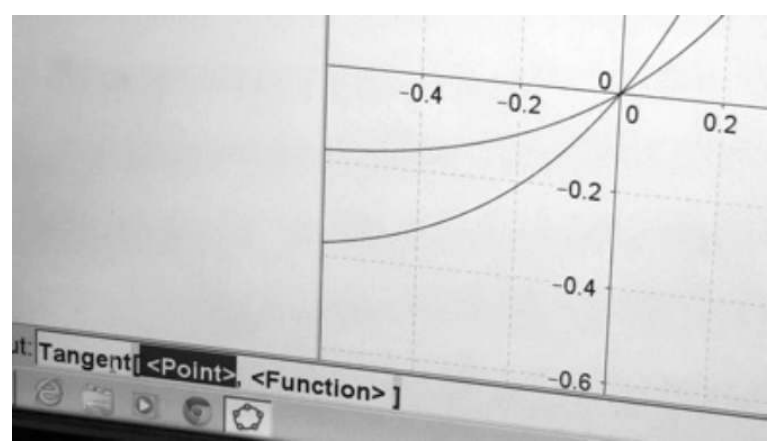

Fig. $6 b$.

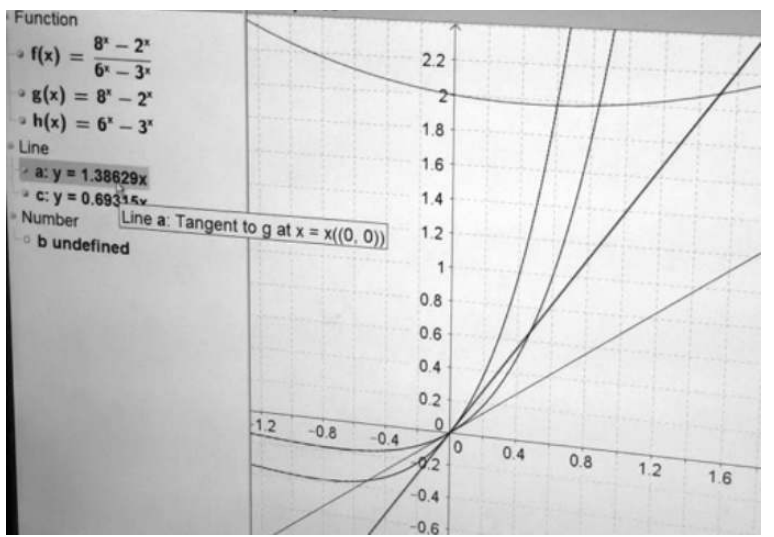

Fig. 6c.

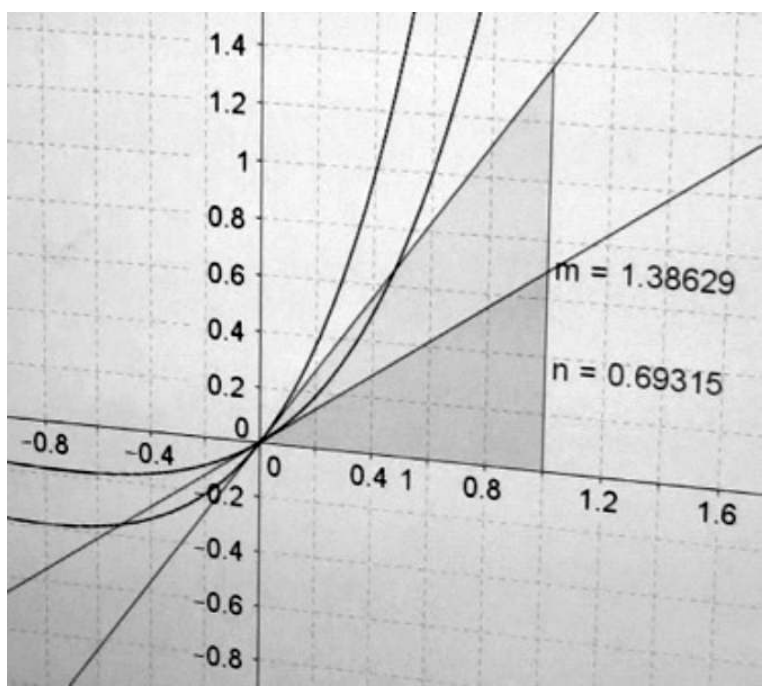

Fig. 6d.

Whereas Kyra and Glen made use of static tangential visualization and successive tangential visualization, respectively, in their demonstrations of the l'Hospital's Rule, constant comparison of these two students' graphical work along with their explanations reveals the fact that their final interpretation differed: while Glen inferred the slope ratio to be 2 by inspection, Kyra actually obtained the same answer by actually typing the slopes ratio $\mathrm{m} / \mathrm{n}$; which was the slight difference between the two students' thinking. After attempting the problem algebraically first (Fig. A5), Macy obtained "a undefined," when she entered the syntax Limit[f,0], a similar strategy that was used by Kyra as well. In an attempt to visualize the l'Hospital's Rule, she suggested graphing the 
numerator and the denominator functions separately, similar to Kyra's thinking. Macy explained, "as $x$ goes to zero, while the original function goes to $2 \ldots$ both of these functions go to zero which is what I expected [highlighting the intersection point (Fig. 7a)]." She concluded her demonstration by typing $(\ln (8)-$ $\ln (2)) /(\ln (6)-\ln (3))$, which appeared as " $\mathrm{c}=2$ " on the algebra sidebar, as a way of checking her work (Fig. 7b).

\section{Figure 7.}

Macy's (a-b) and Sharon's (c-d) work

Fig. 7a.

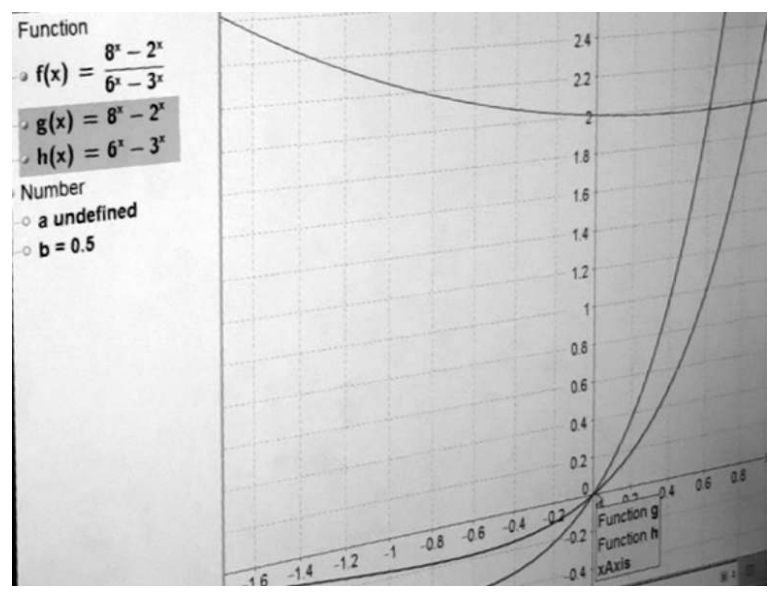

Fig. $7 b$.

\section{Function}

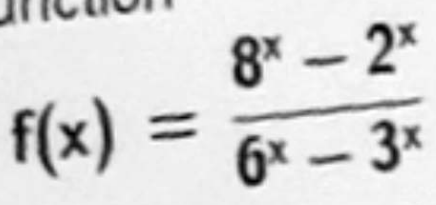

$g(x)=8^{x}-2^{x}$ $h(x)=6^{x}-3^{x}$

Number

a undefined

$b=0.5$

$c=2$

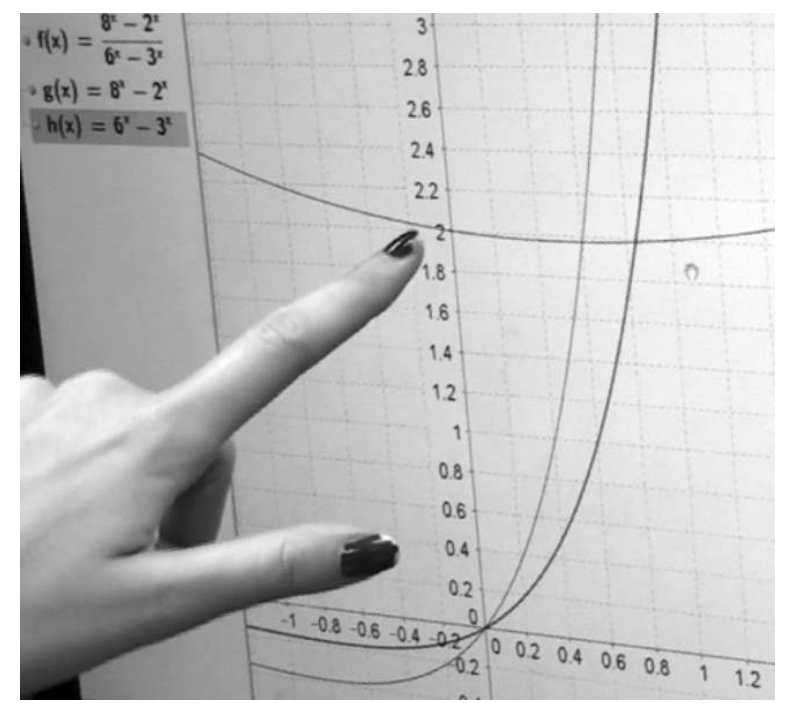

Fig. 7c.

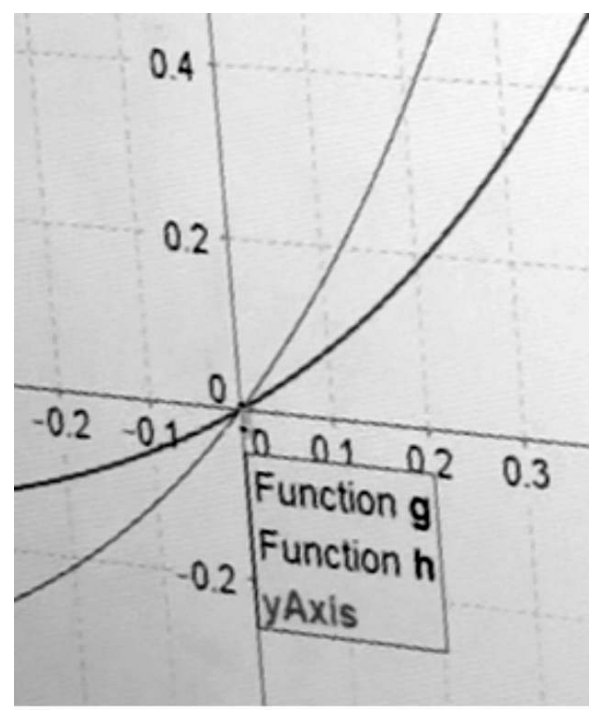

Fig. 7d.

Sharon also followed an approach similar to Kyra and Macy's by which she obtained the graphs of the original function along with the numerator and denominator functions. She explained, "so I did this and the limit is obviously 2 [pointing to the function graph (Fig. 7c)] when I did this algebraically I mean this is gonna be 1-1 over 1-1 which is zero divided by zero so I have to do l'Hospital's so and then I graphed the top and I graphed the bottom [respectively pointing to the graphs of $g$ and $h$ ] and so I tried to figure out what their limit is as $x$ approaches zero 
[highlighting the intersection of $\mathrm{g}$ and h (Fig. 7d)] and I figured that it's gonna be zero over zero so I figured maybe if I do l'Hospital's... so if I do $g^{\prime}$ of zero this is gonna be $\ln (8)-\ln (2)$ and then h' of zero will be $\ln (6)-\ln (3)$ which shows that they are not zero (Fig. A6)". After completing her algebraic derivations, she suggested demonstrating her results by plotting tangent lines on the graphs of $g$ and $h$. While she dragged the cursor towards the Tangents Tool (Fig. 8a), she thought aloud, "is it possible to draw tangent lines at the same time? Maybe a point here [plots an arbitrary point on $g$ ] and this is the tangent line to this function (Fig. 8b)... and a point here [plots an arbitrary point on $\mathrm{h}$ ] and so this is the tangent line to this one (Fig. 8c) I am just zooming out cuz that's really confusing."

Although she was first inclined to plot two tangent lines at the same time, she came up with separately drawn tangent lines, which she later dragged towards the point of interest (Fig. 8d), indicative of successive tangential visualization. She concluded her demonstration by connecting her algebraic and graphical approaches: "I thought this was pretty interesting cuz you know when you do something algebraically or you do it graphically and in this one I kinda incorporated both of them... I mean I graphed the tangent lines and then I didn't really realize yet that the ratio of these two was gonna be 2 but I mean of course it's gonna be 2 because that's what I am doing on paper and like... I never made that connection so it's really cool. So basically from doing it algebraically I figured you do the l'Hospital's once and then because they are not both zero [meaning $g^{\prime}(0)$ and $h^{\prime}(0)$ ] then it'll be able to tell me what the limit is which is 2 . So I mean like... I know that the slopes of the tangent lines are not zero that means I'll be able to find the ratio of these slopes... this one over that one [respectively pointing to the slopes of the tangent lines that appear on the algebra sidebar] is 2 to 1 which is perfect so and then the limit is 2 and that makes sense..."
Figure 8.

Sharon's consequential (successive) tangential visualization

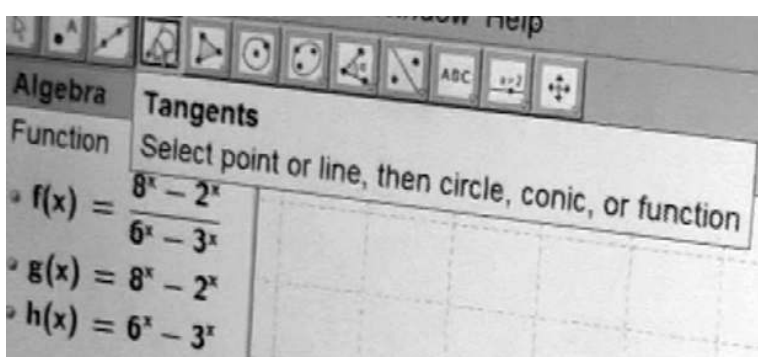

Fig. $8 a$.

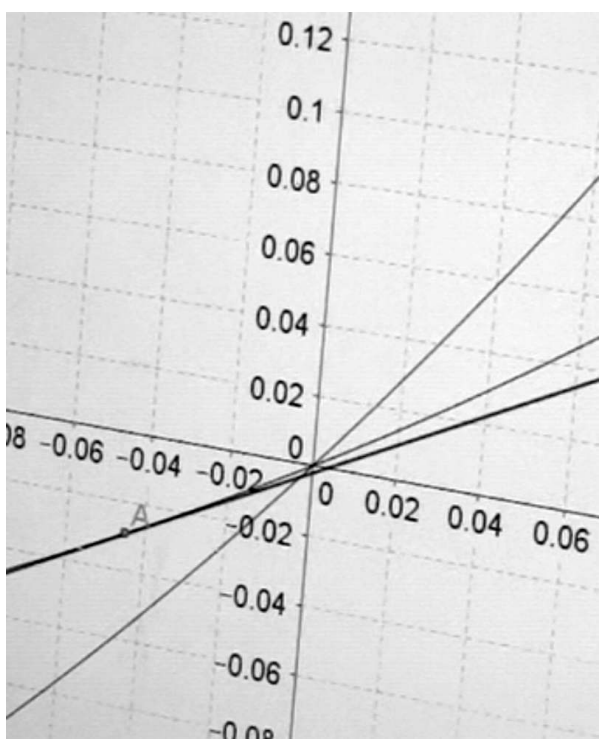

Fig. $8 b$.

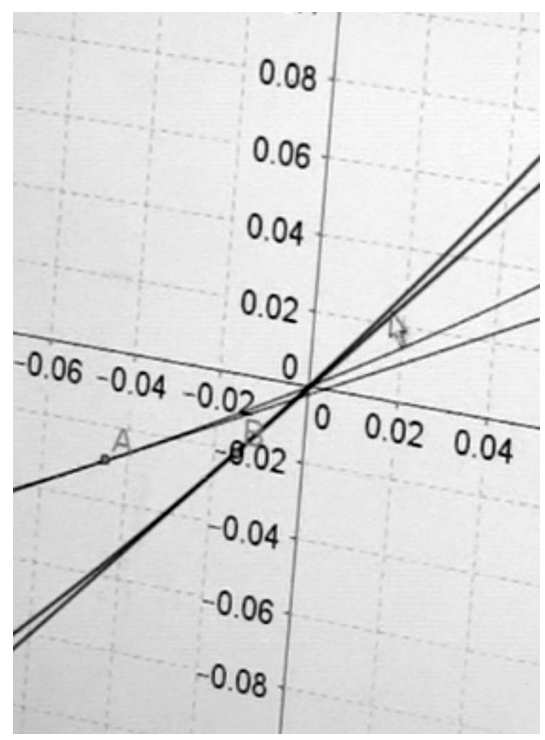

Fig. 8c. 


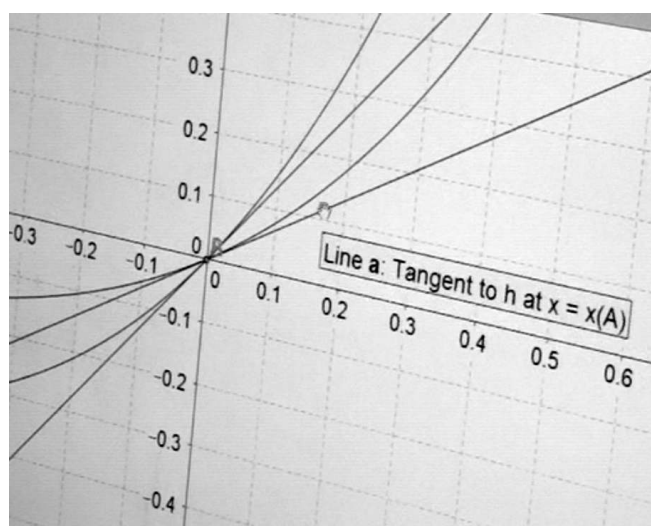

Fig. 8d.

\section{The $0 \cdot \infty$ indeterminate form}

In the problem $\lim _{x \rightarrow 1^{+}} \ln x \tan \frac{\pi x}{2}$ of the $0 \cdot \infty$ form, students who recognized the problem's relevance to the l'Hospital's Rule first rewrote the product as a single guotient by which thev either obtained $\lim _{x \rightarrow 1^{+}}\left(\frac{\ln x}{\cot \frac{\pi x}{2}}\right)$ or $\lim _{x \rightarrow 1^{+}}\left(\frac{\tan \frac{\pi x}{2}}{\frac{1}{\ln x}}\right)$, respectively of the $\frac{0}{0}$ form and the $\frac{\infty}{\infty}$ form. Affter graphing the function via the syntax $f(x)=\ln (x)^{*} \tan \left(\mathrm{pi}^{*} x / 2\right)$, Sharon explained, "I just like to get a big picture about the graph first [zooming out] so here as $x$ approaches 1 ... it's 1 from the right... so I would say that the limit exists at $\mathrm{f}$ of 1 [bi-directional vertical cursor gesture in the direction of the ungraphed $x=1$ line]... but and then I thought it was kinda interesting I just figured to plot in 1 and then $\ln (1)$ is equal to zero [types $\ln (1)$ and $\mathrm{a}=0$ appears on the algebra sidebar] so and then I was kinda wondering why I don't have the point one comma zero on my graph?"

Sharon's explanations triggered more knowledge pieces to emerge, as she provided the undefinedness of the $\tan \frac{\pi x}{2}$ function at $x=1$ as a reason for the function $f$ having a limit at $x=1$. She typed the syntax $f(1)$ and "b = undefined" appeared on the algebra sidebar. She explained, "what if I typed it into the tangent? [types $f(x)=\tan \left(p^{*} x / 2\right)$ which overwrites the previous function] so yeah here it's undefined [recognizes the vertical asymptote at $x=1$ ] .. can I go back to my other one? [selects undo and brings the previous function graph back]... So I mean I am not really actually too sure... So the limit as $x$ approaches $1 \ldots$ When I typed $f(1)$ it interprets as zero times undefined so I guess then $f(1)$ does not exist even though it's on my graph [plots a point on the function graph and drags it towards the point of interest] it changes at every slide so the limit does exist [pointing to the $y$-coordinate of Point $\mathrm{A}$ ] and I would say an irrational number cuz if I plug in 1 it's undefined." Macy and Lindsey followed a similar approach as Sharon's by plotting a draggable point on the function graph, which helped them give an estimate for the limit value as -0.64 (Fig. 9a-b). In Glen's case, the point on the function graph resulted in $A(1,-0.64)$ that appeared on the algebra sidebar (Fig. 9c); Glen noted that it shouldn't have happened as the induced function is not defined at $x=1$. As he explained, "Maybe graphically you can't really get the exact value but you get a pretty close approximation."

Figure 9.

Macy (a), Lindsey (b) and Glen's (c) work

Function
$\boldsymbol{f}(\boldsymbol{x})=\ln (\boldsymbol{x}) \tan \left(\pi \frac{\mathbf{x}}{\mathbf{2}}\right)$
Point
$-\mathrm{A}=(0.99,-0.64)$

Fig. 9a.

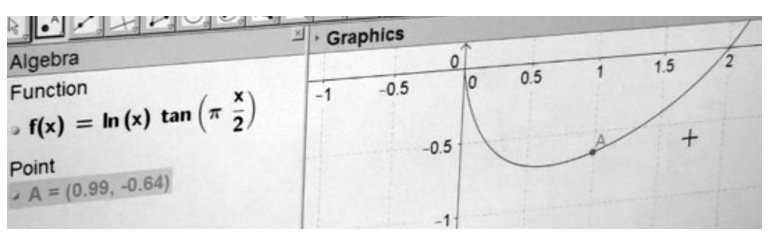

Fig. 9b.

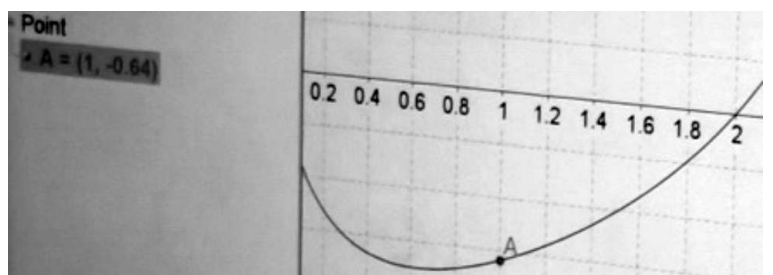

Fig. 9c. 
Sharon's thought process stimulated her willingness to solve the problem algebraically in an attempt to provide an explanation for this happenstance. She did not realize the problem's connection to the l'Hospital's Rule at first. Out of the eight students, only Gilda, Sharon, and Kyra indicated the problem's relevance to the l'Hospital's Rule by actually algebraically solving the problem, who also provided an exact solution for the limit. The other students simply offered an estimate for the limit value from the graph of the function $f(x)=\ln (x)^{*} \tan \left(\mathrm{pi}^{*} x / 2\right)$ they typed. After graphing the function via the syntax $g(x)=\ln (x)^{*} \tan \left(\mathrm{pi}^{*} x / 2\right)$, Kyra came up with a syntax of her own by typing the syntax Limit $[g, 1]$ and the value $b=0$ appeared on the algebra sidebar (Fig. 10a). Kyra explained, “it gave me zero but it shouldn't be zero according to the graph it's negative [zooming into the graph (Fig. 10b)]." She then decided to solve the problem algebraically in order to find an exact solution (Fig. A7), which, she verified using the software by typing the syntax $-2 / p i$, which appeared as $b=-0.64$ on the algebra sidebar (Fig. 10c). Gilda followed a similar approach as Lindsey and Sharon's. Gilda not only plotted a draggable point to obtain an estimate for the limit value, she also came up with a syntax of her own by typing $(1, \mathrm{~g}(1))$, which resulted in "E undefined" on the algebra sidebar. Gilda explained, "it shows it as continuous on the graph and it shouldn't (Fig. 10d)."

Figure 10.

Kyra (a-c) and Gilda's (d) work

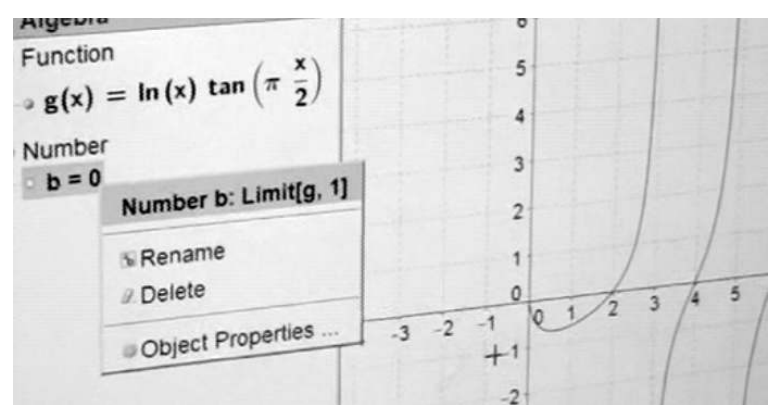

Fig. 10a.

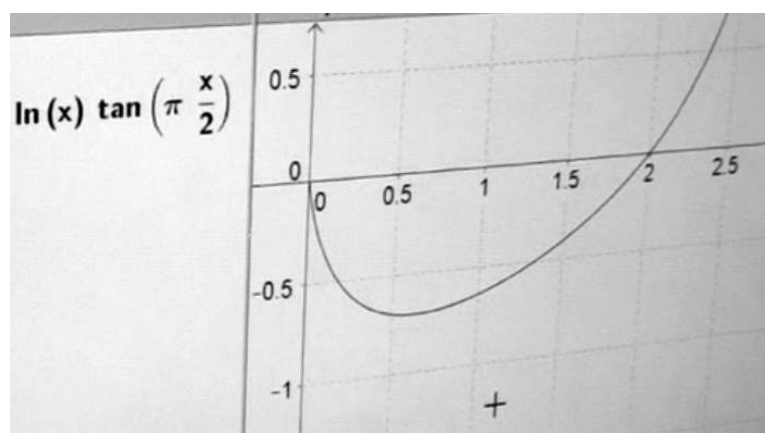

Fig. $10 b$.

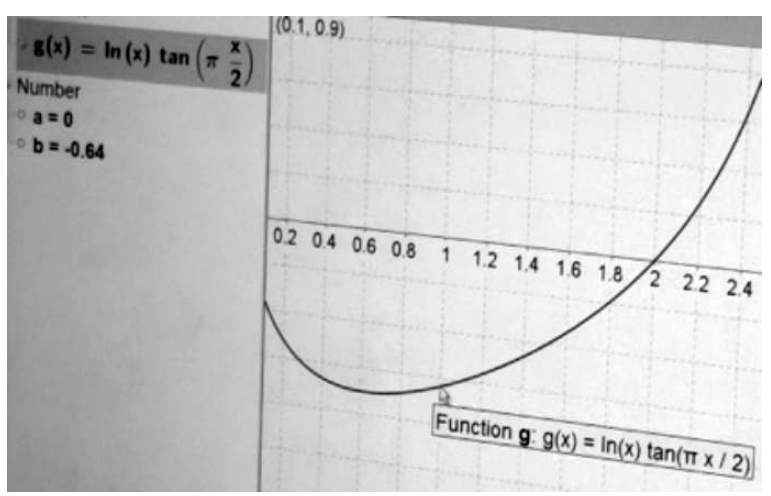

Fig. 10c.

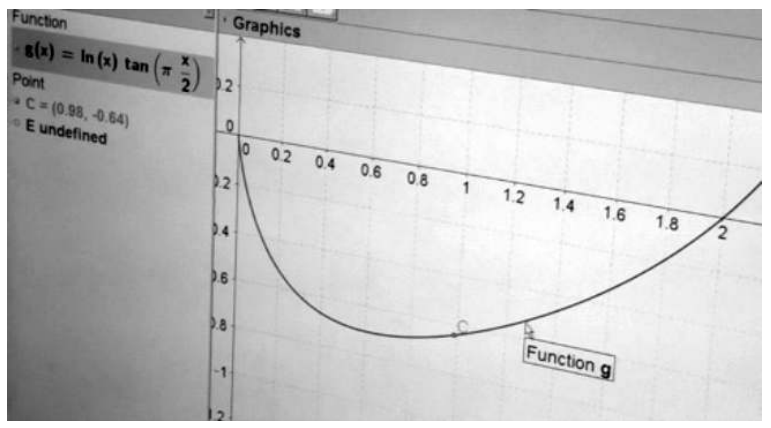

Fig. 10d.

After solving the limit problem algebraically, Sharon illustrated the l'Hospital's Rule graphically by actually graphing the numerator and the denominator functions, $f(x)=\ln x$ and $(x)=\cot \frac{\pi x}{2}$, respectively. For that purpose, she first plotted an arbitrary point A on the function graph $h(x)=\frac{f(x)}{g(x)}$, and she explained, "I would want the point right here and then also right here [pointing on the graph of $f$ and $g$ respectively in a vertical direction] I mean a vertical line will go through all three of them [types $x=x(A)$ 
and obtains a vertical line passing through A]." She then used the Intersect Two Objects Tool to plot the intersection points B and C, respectively, on the function graphs $f$ and $g$ (Fig. 11a). Sharon then explained, "so then I will find the limit of this one and then the limit of that one [respectively pointing to the graphs of $f$ and $g$ while dragging point A towards $x=1$ ] so when A approaches 1 from the left then $B$ and $C$ both approach 1 as well [she remarks that A disappears when $x=1$ ] when it's equal to 1 then it goes away and then greater than 1 , A appears again." After hiding the vertical line, she constructed tangent lines on the functions $f$ and $g$, respectively, by using the Tangents Tool (Fig. 11b). She explained, "because right here the derivative at this point [pointing to $\mathrm{B}$ ] would be the slope of this tangent line [pointing to the tangent line] so and then I would like to move A... so and then right here the slope of this tangent line at point B is gonna be the derivative so and then as I move over to the point right here [drags point A towards $x=1$ (Fig. 11c)] then the slope of the tangent line right there at $B$ is going to be equal to the derivative of this function [pointing to $f$ ] at this point [pointing to B] and then the same thing for the other which is the slope right here of this tangent line [highlighting the tangent line passing through C (Fig. 11d)]. So the limit of this one as it approaches 1 [pointing to $\mathrm{A}$ ] is going to be the same as the quotient of the derivatives... or as quotient of the slopes of these two lines..."
Figure 11.

Sharon's simultaneous tangential visualization

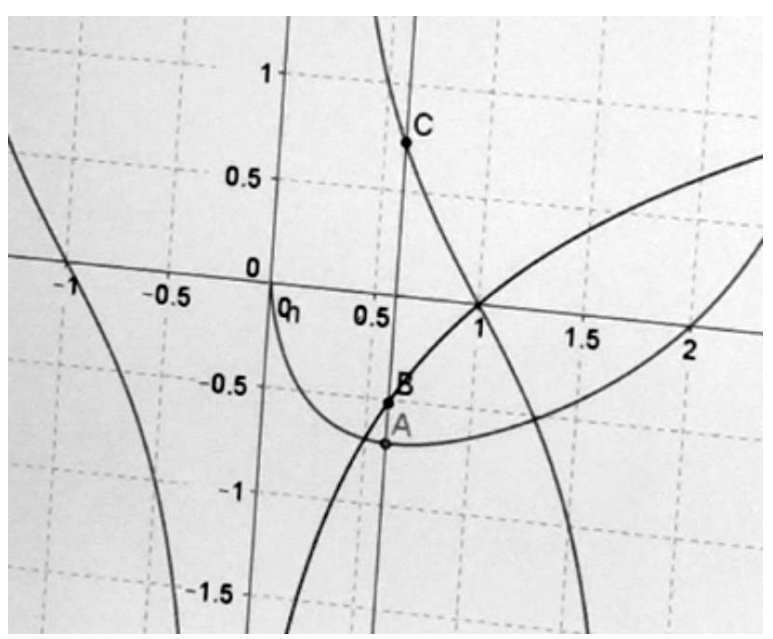

Fig. 11a.

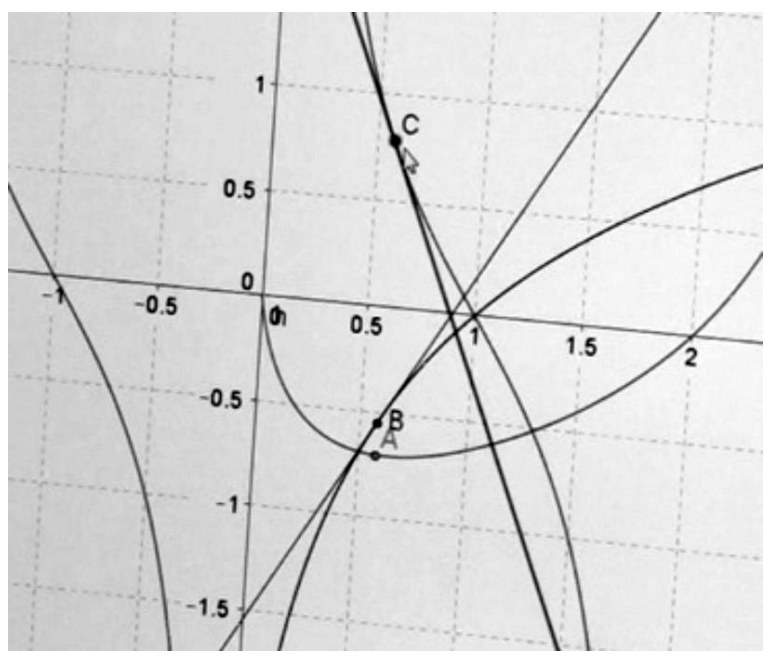

Fig. $11 b$.

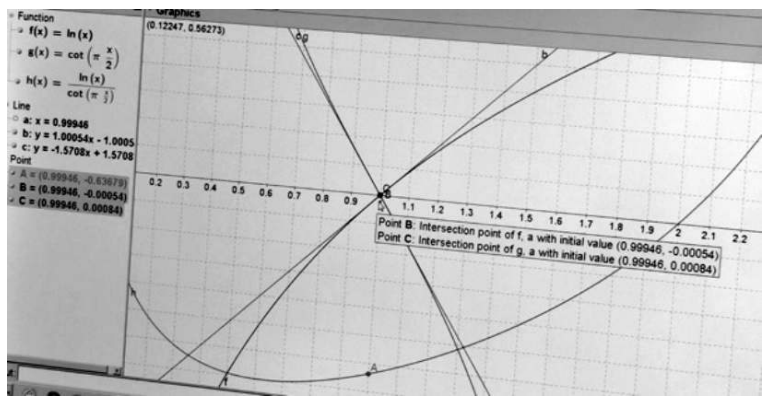

Fig. 11c. 
Gunhan Caglayan

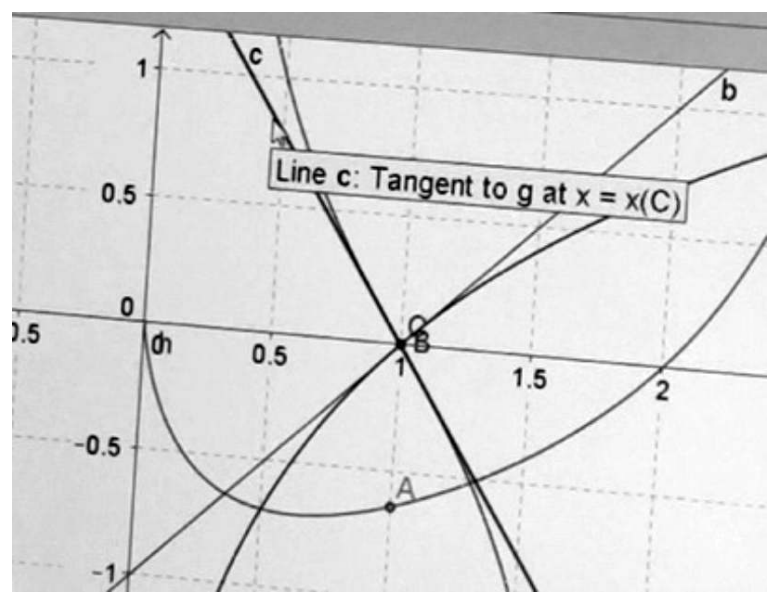

Fig. 11d.

As outlined above, Sharon's visualization could be categorized as of simultaneous tangential type as she sought for the limit value by observing the behaviors of the tangent lines changing at the same time when she dragged Point A on the original function graph. For the same problem, after solving the problem algebraically, during which she went through some hesitations as to which function to represent her numerator function (Figs. A9-A11); Gilda wanted to revise her demonstration for the purpose of illustrating the l'Hospital's Rule graphically. For that purpose, she graphed the numerator and the denominator functions, $f(x)=\ln x$ and $g(x)=\cot \frac{\pi x}{2}$, separately, similar to Sharon's thinking, and then graphed the quotient function $h(x)=\frac{f(x)}{g(x)}$. The slight difference between these two students' visualizations was in that Gilda did not plot any point on the quotient function graph; instead, she graphed two arbitrary points on each of the graphs of $f$ and $g$. She explained, "ok derivative... derivative is what we use when we do the l'Hospital's Rule... so and then we are taking at a point... the tangent of both curves at point $1 \ldots$ [pointing to the desired point of tangency (Fig. 12a)] so the slope of the tangent line gives you the derivative [constructs tangent lines on each curve (Fig. 12b)] and then where the tangent lines intersect that will give you the point... so I want them to intersect at 1 [drags point A towards $x=1$ (Fig. 12c)] because that's the question... I want to know what the limit is at that point [drags point B towards $x=1$ (Fig. 12d)].

Figure 12.

Gilda's successive tangential visualization

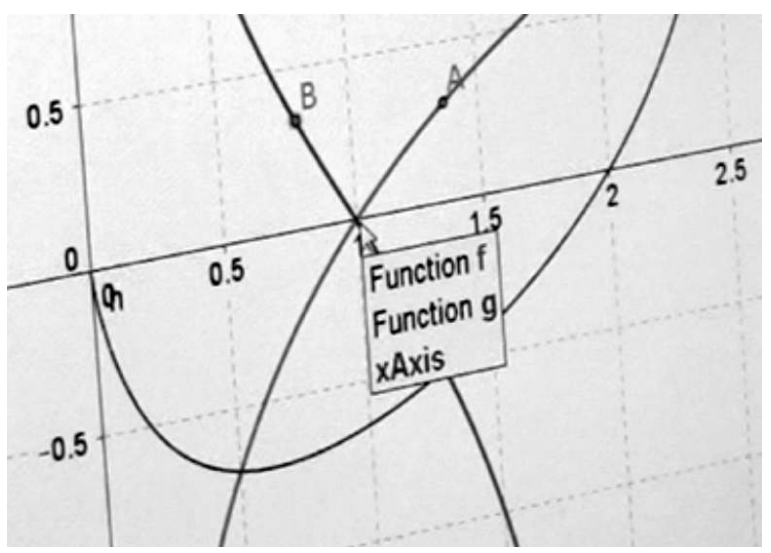

Fig. 12a.

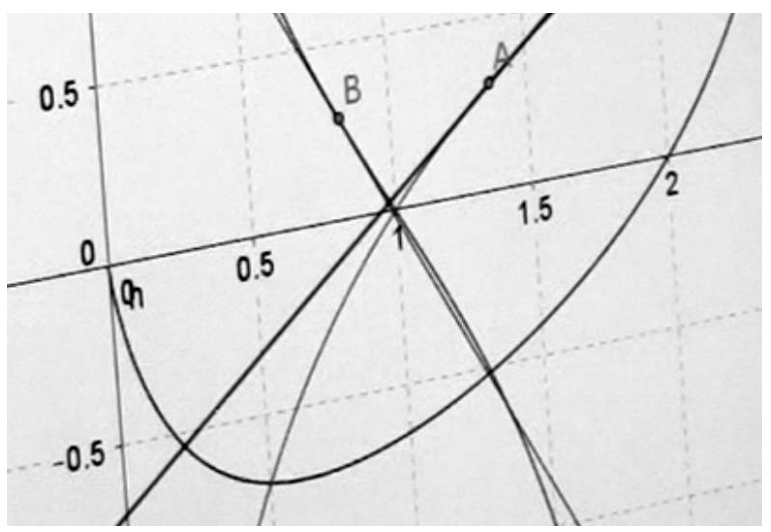

Fig. $12 b$.

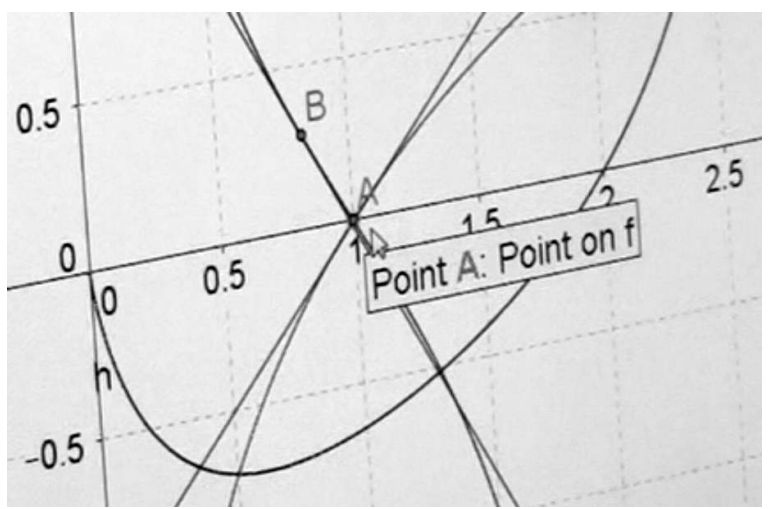

Fig. 12c. 


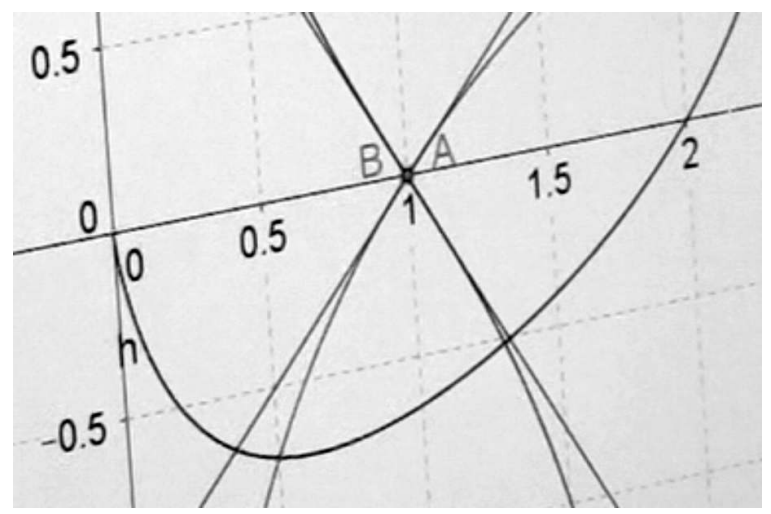

Fig. 12d.

When the interviewer probed on why she did what she did, Gilda responded, "the derivative of the top function... so the derivative of $\ln$ of $x$ at 1 is just $1 \ldots$ and the derivative of the bottom function would be... the derivative of cotangent pi $\mathrm{x}$ over two which is negative pi over two cosecant square pi $x$ over two so then you get negative pi over two... so this makes sense [very excitedly] so it's one [pointing to the slope of the tangent line $y=x-1$ on the algebra sidebar] over negative pi over two [pointing to the slope of the tangent line $y=-1.5708 x+1.5708$ on the algebra sidebar] well I'm guessing -1.5708 is negative pi over two... so one over negative pi over two and then it gives you negative two over pi [very excitedly] which is the limit... this is pretty cool like seeing how it relates to the graph you know l'Hospital's Rule was something too abstract to me... you know you are able to do it because you know the formula but it's cool to see it what it really means on a graph." Gilda's method could be thought of as indicative of successive tangential visualization as she separately dragged the tangent lines towards the point of interest. It is also worth noting Gilda's realization "where the tangent lines intersect that will give you the point" as indicative of meta-representational competence in her visualization of the separately constructed tangent lines for a specific purpose; that is, for the purpose of deducing the desired common point of tangency for both function graphs.
The $\infty-\infty$ indeterminate form

In problems $\lim _{x \rightarrow 1^{+}}\left(\frac{x}{x-1}-\frac{1}{\ln x}\right)$ and $\lim _{x \rightarrow 0^{+}}\left(\frac{1}{x}-\frac{1}{e^{x}-1}\right)$ of the $\infty-\infty$ form, students first rewrote the difference as a single quotient in each case by which they obtained $\lim _{x \rightarrow 1^{+}}\left(\frac{x \ln x-x+1}{(x-1) \ln x}\right)$ and $\lim _{x \rightarrow 0^{+}}\left(\frac{e^{x}-1-x}{x\left(e^{x}-1\right)}\right)$, respectively, each of the form. In her demonstration of the first problem, after plotting the induced function graph, Kyra used the Limit[f,1] syntax to obtain the desired limit value, which appeared as $a=0.5$ on the algebra sidebar (Fig. 13a); which caused Kyra's willingness to solve the problem algebraically (Fig. A12). She explained, "at first, combine into one fraction so you can see if there is something that can factor out and simplify it." In the second step of applying the l'Hospital's Rule, Kyra commented, "when you put 1 in here $\ln (1)$ is zero so that [pointing to the quotient $\left.\frac{\ln (1)}{\ln (1)+1-(1)}\right]$ would still be zero over zero so then I tried doing the l'Hospital again so then I came up with this and factored out the $x^{-1}$ [pointing to the quotient $\left.\frac{x^{-1}}{x^{-1}\left(1+x^{-1}\right)}\right]$ so that gives me one over one plus $x$ to the negative one... and in that case when I substitute 1, I get one half (Fig. A12)."

Upon the interviewer's probing whether she was expecting one half as her answer, Kyra responded that the l'Hospital's Rule could be visualized by noting that all the quotient functions (i.e. $x_{x-1}$ the quotient $\frac{x \ln x-x+1}{(x-1) \ln x}$ and the quotient $\left.\frac{x^{-1}}{x^{-1}\left(1+x^{-1}\right)}\right)$ at each stage would behave similarly near the same point of discontinuity at $x=1$. She explained, "at first, when the function is split up like this [pointing to the graph of $f$ ] then it comes to infinity minus infinity... it's just a completely different graph compared to both of them [meaning the $\frac{x}{x-1}$ and $\frac{1}{\ln x}$ ] individually because $x$ over $x$-1 and 1 over $\ln$ of $x$ have similar looking graphs and then when you put the two of them together it makes this graph [pointing to the graph of f] instead." She illustrated her idea by 
graphing the quotient function at the second stage, indicative of derivative functional (static) visualization (Fig. 13b). She further commented that she would get a similar behavior in the vicinity of $x=1$ had she graphed the quotient function at the third stage. Macy graphed the numerator $x \ln x-x+1$ and the denominator $(x-1) \ln x$ of the simplified quotient $\frac{x \ln x-x+1}{(x-1) \ln x}$ separately in an attempt to demonstrate why she would need to use the l'Hospital's Rule in this problem (Fig. 13c). As she explained, "as $x$ goes to 1 both functions the top and the bottom go to zero [pointing to the $\mathrm{x}$-intercept $(1,0)]$ so it's of the form zero over zero which is why you have to do the l'Hospital's, well in this case twice."

Figure 13.

Kyra's (a-b) and Macy's (c) visualization

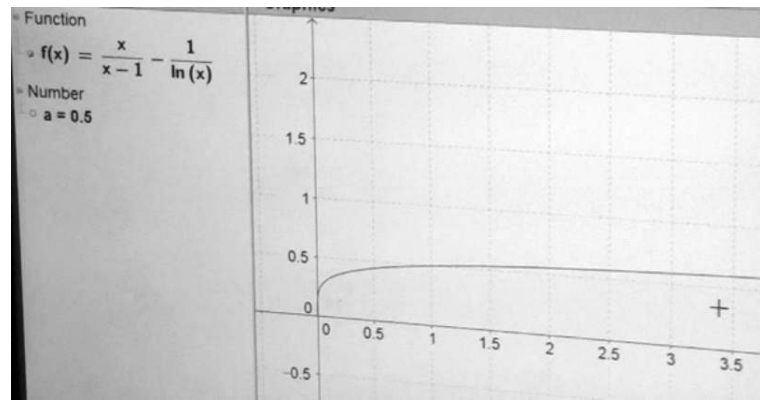

Fig. 13a.

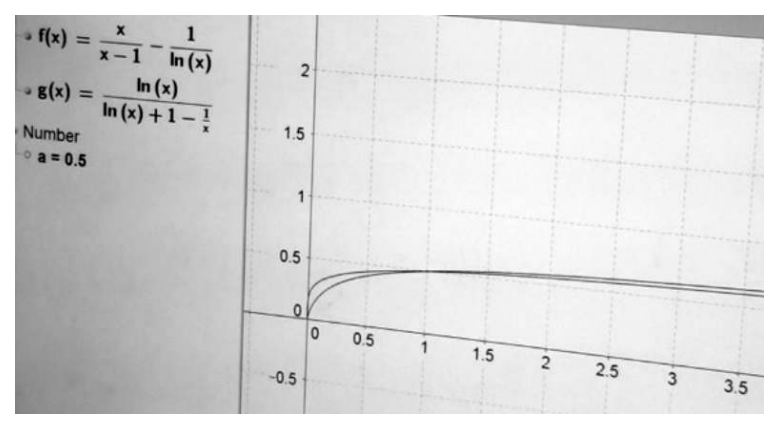

Fig. $13 b$.

In a manner similar to Macy's, upon separately graphing the numerator $x \ln x-x+1$ and the denominator $(x-1) \ln x$ of the simplified quotient $\frac{x \ln x-x+1}{(x-1) \ln x}$, Sharon followed her simultaneous

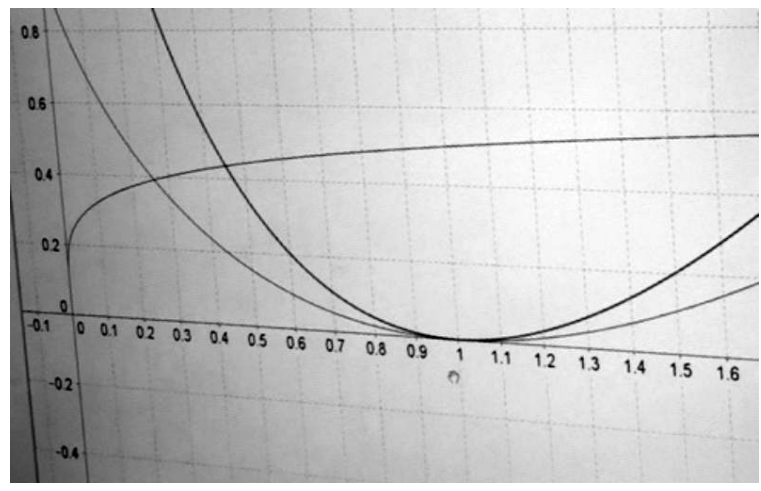

Fig. 13c.

tangential visualization strategy (Fig. 14a) and remarked "the derivatives are undefined at that point... at $x=1$ " when she dragged A towards $x=1$ and observed that all there points A, B, C along with the two tangent lines disappeared as Points $\mathrm{B}$ and C were dependent on Point A in Sharon's original construction (Fig. 14b). She further remarked, "it makes sense that if they are undefined for $B$ and $C$ so they would also be undefined for A... so now why are they all undefined... so the derivative of these two functions at $x=1$ would be undefined if the slope of tangent line was undefined."

Figure 14.

Sharon's simultaneous tangential visualization in a 2-step problem

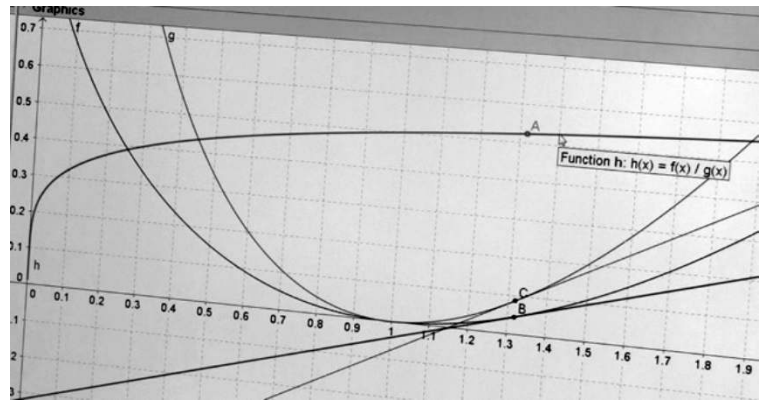

Fig. 14a.

Sharon then realized that it was a 2-step problem and graphed the derivative functions corresponding to $f(x)=x \ln x-x+1$ and $g(x)=(x-1) \ln x$ after hiding the graphs of $f$ and $g$. She demonstrated her simultaneous tangential visualization this time by 
plotting points $\mathrm{B}$ and $\mathrm{C}$ on the derivative functions $f^{\prime}(x)$ and $g^{\prime}(x)$, respectively:

"So and then because l'Hospital's Rule didn't work the first time then you had to take the derivative and try it again so now what I'm doing is I am plotting the derivatives and then I am seeing if I could find what the limit of this is [pointing to Point A] using the slopes of the derivatives [pointing to the derivative function graphs respectively]... so now I'm gonna make the tangent lines [constructs tangent lines on the derivative function graphs and drags Point A towards $x=1$ (Fig. 14c)] so now I wanna see that if you take the slopes of the tangent lines at $x=1$ if those slopes would be defined [drags Point A to $x=1$ and remarks that all three points and the two tangent lines disappears] so here the slope of this tangent line and the slope of this tangent line are undefined."

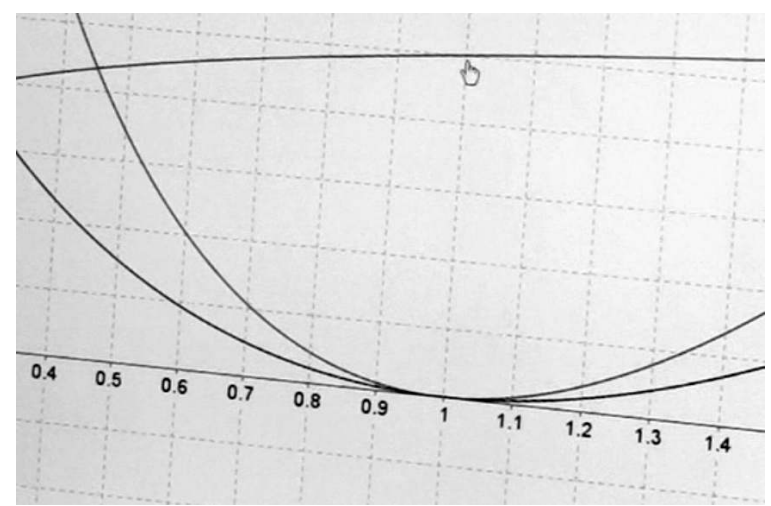

Fig. 14b.

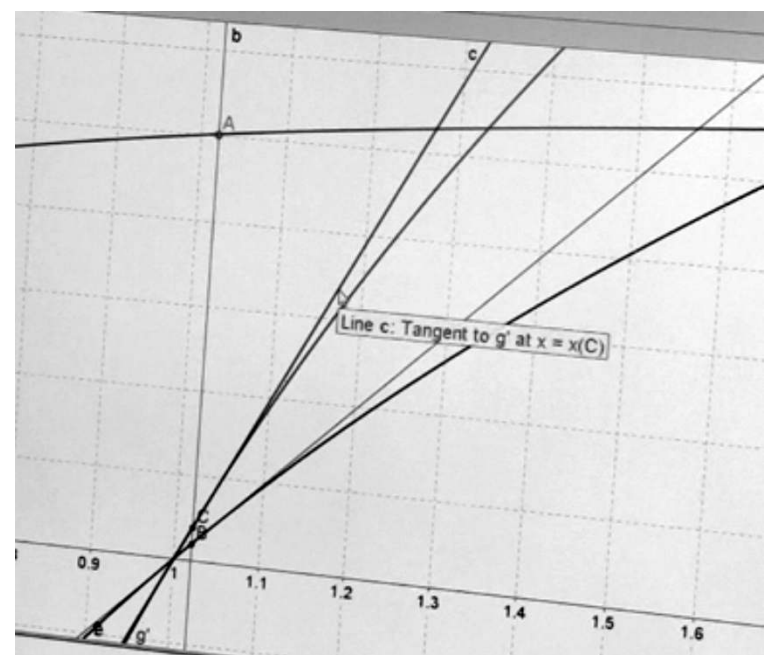

Fig. $14 c$.

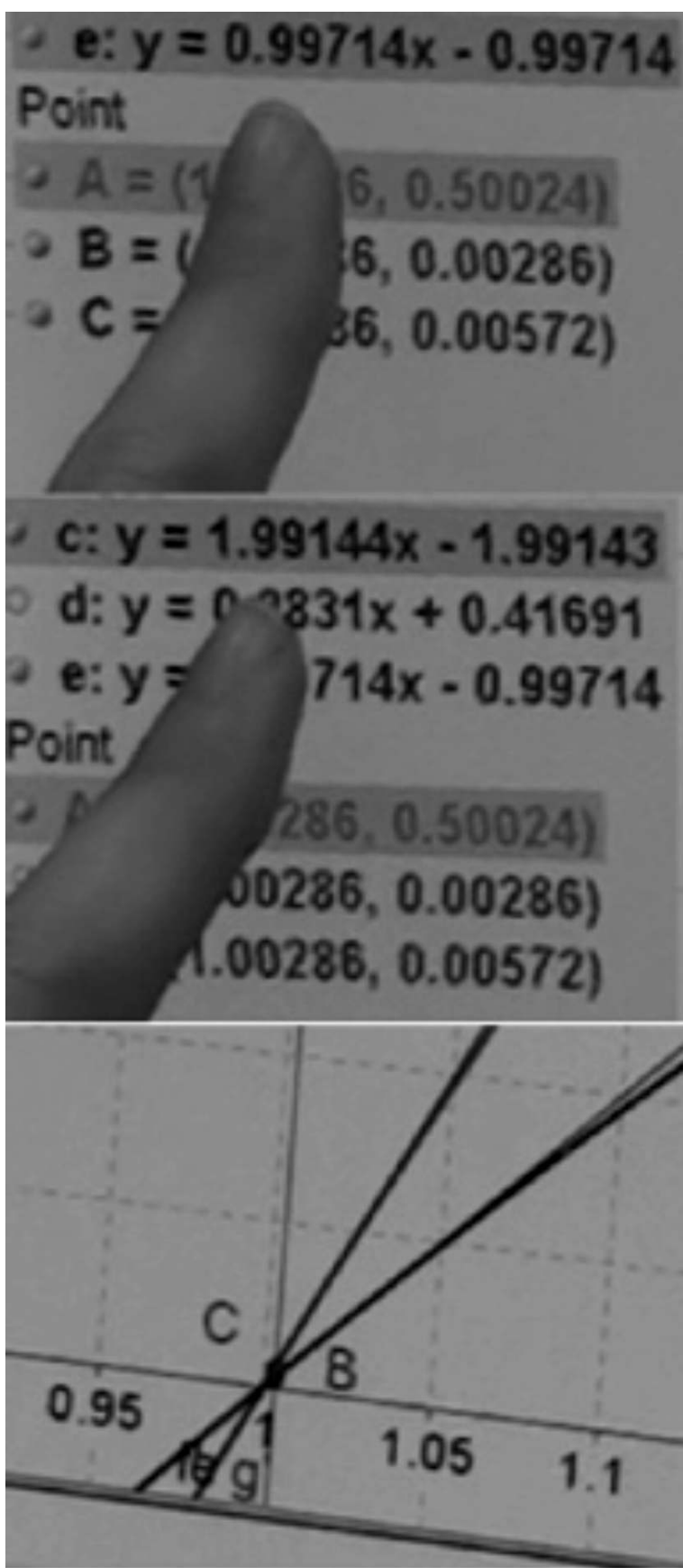

Fig. $14 d$.

She concluded her demonstration by explaining how she would relate the slope of the tangent line with the second derivatives as "so this one is 0.99 so this is the slope of the tangent line of this function [pointing to the graph of $f^{\prime}$ ] at the point $x=1 \ldots$ well approximately cuz we are a little bit off of $x=1$ and then this one right here which is 1.99 is the slope of 
the tangent line of this graph which is $g^{\prime}$ at the point $x=1 \ldots$ so this right here is the second derivative of $f$ [pointing to the slope of the tangent line on $f^{\prime}$ ] at $x=1$ and then this one right here is the second derivative of $g$ [pointing to the slope of the tangent line on $g^{\prime}$ ] when $x$ is equal to $1 \ldots$ about 1 (Fig. 14d)."

Whereas Sharon's visualization strategy could be categorized as of simultaneous (coherent) tangential type, Gilda's strategy slightly differed from Sharon's in that Gilda made use of a common tangency point, which yields her visualization to be of static tangential type. For that purpose, after writing the difference as a quotient, Gilda first graphed the functions $f(x)=x \ln x-x+1$ and $g(x)=(x-1) \ln x$ along with the corresponding derivative functions, respectively; hid the graphs of $f$ and $g$; and used her "derivative functions intersect at $x=1$ " argument to set the stage for her visualization of the l'Hospital's Rule (Fig. 15a). She then used the Intersect Two Objects Tool to plot the intersection point A. Upon the interviewer's probing why she hid the graphs of $f$ and $g$, Gilda explained, "in this case like then... you have a problem like the previous one... that their tangent lines would be the same horizontal line $y=0$ for both and it'll be a problem because... it's about because you need the two tangent lines... oh! because it gave me two tangent lines both $y=0$ [pointing to the function graphs $\mathrm{f}$ and $\mathrm{g}$ that are tangent to each other (Fig. 15b)] so you get zero over zero and you can't get an answer with zero over zero."

Gilda further explained that she would not be able to use her tangent line approach with the graphs of functions $f$ and $g$, instead, as she explained, "so instead of doing the tangent line approach which we cannot with the actual functions $f$ and $g . .$. we do it with the derivative functions so here $f^{\prime}$ and this is $g^{\prime}$ and they intersect at one comma zero [highlights point A (Fig. 15c)] so I graph the tangents to the $f^{\prime}$ and the $g$ ' so then I know it's gonna be a ratio... ratio of the slopes of the tangent lines and they are actually
Figure 15.

Gilda's static tangential visualization in a 2-step problem

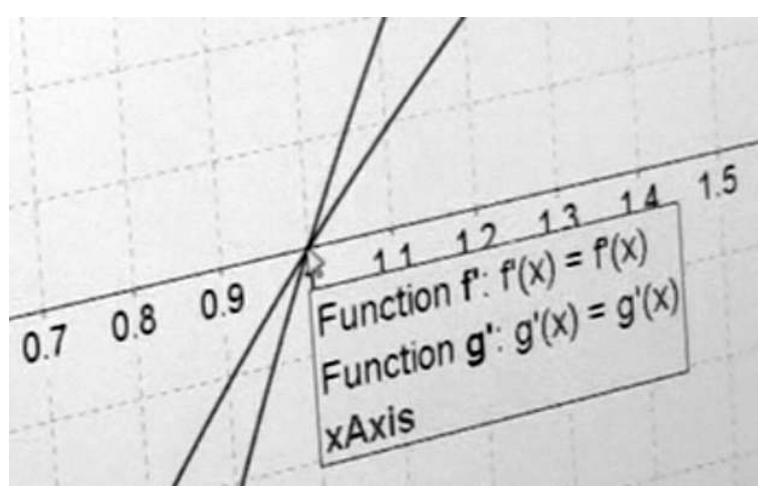

Fig. $15 a$.

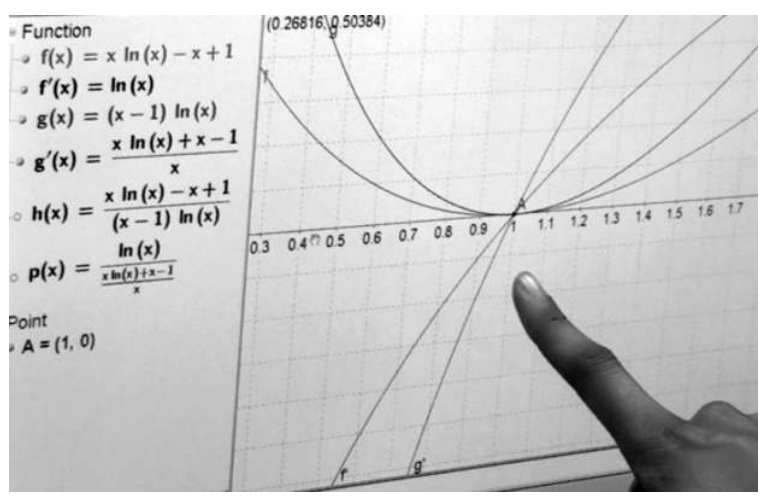

Fig. $15 b$.

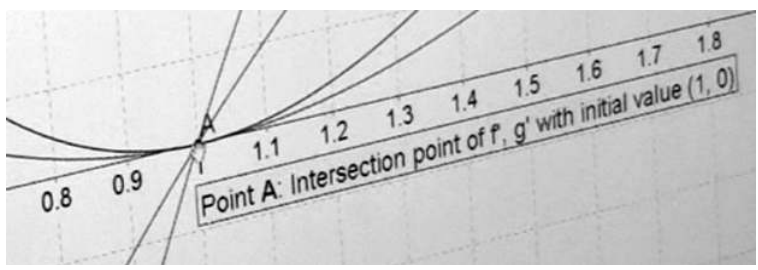

Fig. 15c.

lines and my a is the tangent to $f^{\prime}$ and $\mathrm{b}$ goes with $g^{\prime}$ so the ratio is one half [respectively highlighting to the slopes of $y=1 x-1$ and $y=2 x-2$ that appear on the algebra sidebar (Fig. 16a)]. 
Figure 16.

Gilda's static tangential visualization (cont.)

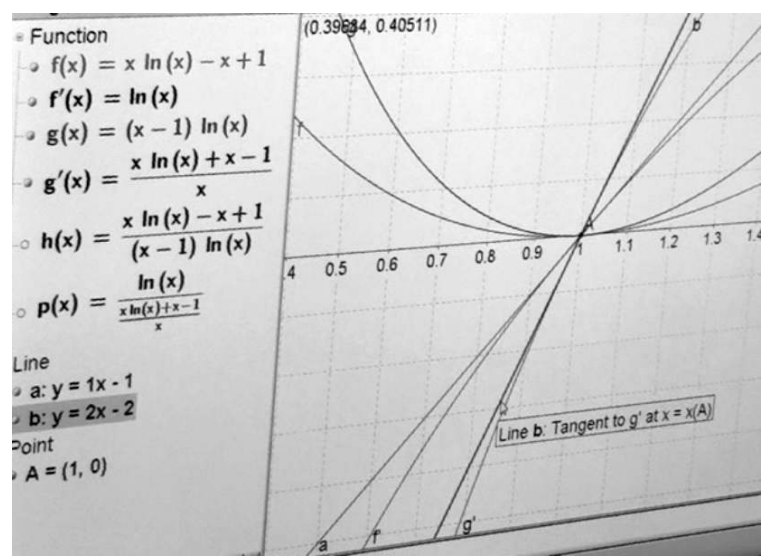

Fig. $16 a$.

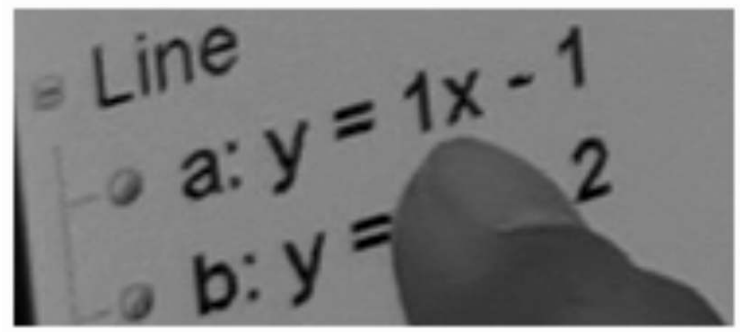

Fig. $16 b$.

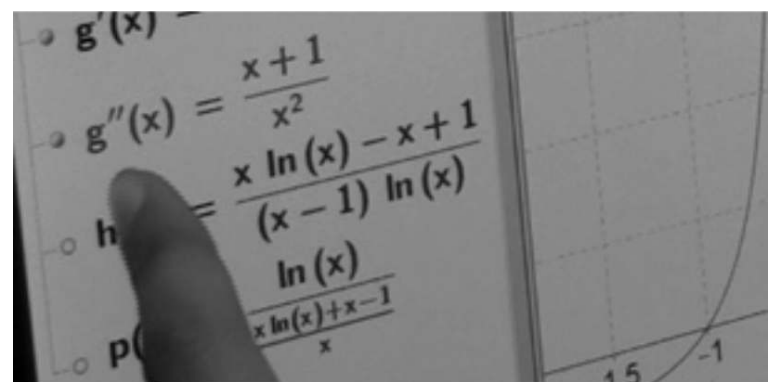

Fig. $16 c$.

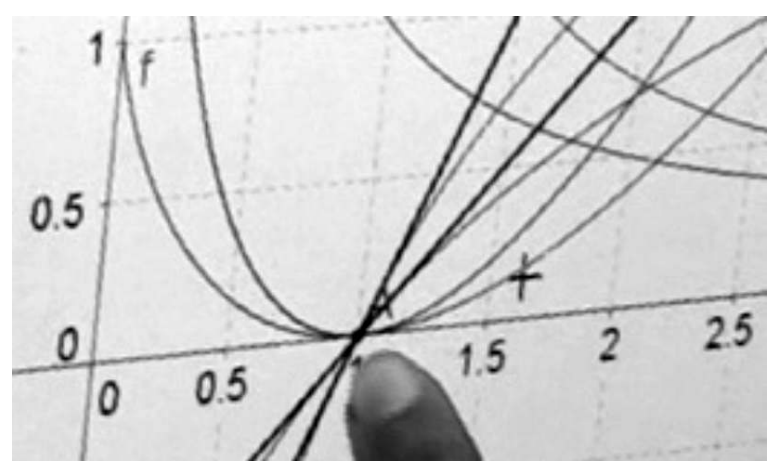

Fig. 16d.

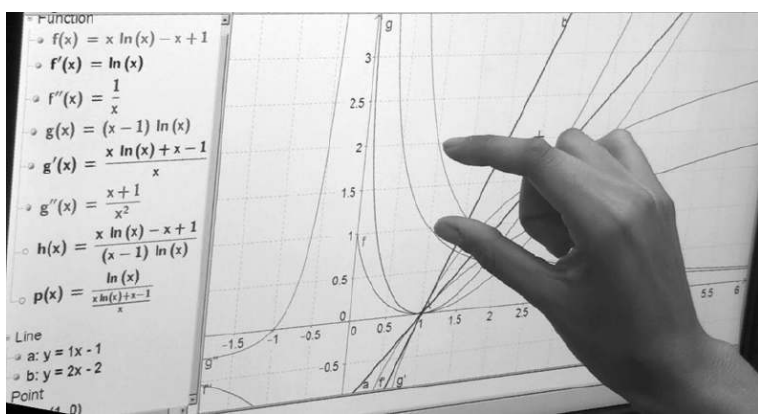

Fig. 16e.

Gilda concluded her demonstration by providing further insights by actually graphing the second derivatives, $f^{\prime \prime}$ and $g^{\prime \prime}$, and evaluating them at $x=1$, respectively, as another way of asserting that the answer to the original problem would be the ratio of $f^{\prime \prime}(1)$ to $g^{\prime \prime}(1)$. She explained, "so the slope of line a is 1 [pointing to the slope of line $y=1 x-1$ on the algebra sidebar (Fig. 16b)] which is the $y$-value of $f^{\prime \prime}$ at $x=1$ and then the slope of line $\mathrm{b}$ is 2 [pointing to the slope of line $y=2 x-2$ on the algebra sidebar] which is the y-value of the $g^{\prime \prime}$ [pointing to the $g^{\prime \prime}$ equation on the algebra sidebar (Fig. 16c)] at $x=1$ and there is the ratio again 1 over 2 [thumb-index finger gesture demonstrating the 1:2 ratio on the graphs of $f^{\prime \prime}$ and $g^{\prime \prime}$ (Fig. 16e)] so the 1 to 2 ratio comes up in many ways."

At the end of her demonstrations, Gilda brought an interesting discussion to the table. She posed, "so for example if these two curves like [pointing to $f$ and $g$ ] were say... same thing but all shifted up like 1 unit so the tangent line would be $y=1$ right? and then you'd still have a problem with the limit as $x$ goes to 1 ? Would the ratio still be undefined?" When the interviewer probed on Gilda's question by responding, "what are your thoughts about that?" Gilda explained, "so the slope is still zero because it is a horizontal line I thought for a second that it was because it's on the $x$-axis [pointing to the $x$-intercepts of the original functions $f$ and $g$ (Fig. 16d)] but now I realize it's because it's [meaning the tangent lines] a horizontal line so it doesn't matter whether it's on 
Gunhan Caglayan

the axis or not... so now I realize it's because it's a horizontal line so regardless of where it is on the graph, because it's a horizontal line that's what makes the ratio undefined."

Although she did not test her conjecture, it was important to come up with a conjecture like this one, which helped Gilda strengthen her reasoning about the l'Hospital's Rule in a particular situation where the two original functions were tangent to each other with the same horizontal tangent line at the common point of tangency. As she further explained, "like here if the tangent line wasn't a horizontal line then I would have been able to get the ratio just using the slopes of the tangent lines of $f(x)$ and $g(x) \ldots$ but I couldn't do that here instead I had to work with the tangent lines of derivative functions $f^{\prime}$ and $g^{\prime} \ldots$ and doing the $f^{\prime}$ and $g^{\prime}$ I was able to find that ratio... and even if that wouldn't have worked, for example, then doing the $f^{\prime \prime}$ and $g^{\prime \prime}$ we could also find that ratio and I'm guessing that could go on forever until you find that ratio." Gilda, in a sense, offered an algorithm for multi-step l'Hospital's problem in general with reference to the slopes of the tangent lines of the numerator and denominator functions, at each step.

\section{DISCUSSION AND CONCLUSIONS}

\section{A Summative Look at Math Majors' Knowledge of the L'Hospital's Rule}

This section summarizes the findings above framed in the meta-representational competence (diSessa, 1988, 2004) and visualization of derivatives (Artigue, 1991; Hughes-Hallet, 1991; Zimmermann, 1991) perspectives in the context of the representation of the l'Hospital's Rule in a multi-representational dynamic environment. Math major participants of the present report came up with various inventions and designs of new representations (MRCl) in their visualizations of the l'Hospital's Rule: (i) Static tangential visualization in exploring limit problems of the 0 / 0 form (Kyra); (ii) Consequential (successive) tangential visualization in exploring limit problems of the 0 / 0 form (Sharon, Glen); (iii) Local-tangential and derivative functional (static) visualizations in exploring limit problems of the $0 / 0$ form (Andy); (iv) Limit[f,a] syntax (Anna, Kyra, Macy); (v) $y(B) / y(A)$ syntax in derivative-functional (dynamic) visualization (Gilda); (vi) Retrieving tangent line's slope via slope measurement tool (Kyra, Glen, Sharon); (vii) Visualizing the l'Hospital's Rule (in limit problems of 0 / 0 type) as the ratio of (a) the derivatives of the numerator and denominator functions (Andy, Sharon); (b) slopes of the [tangent] lines (Glen, Kyra, Sharon); (viii) All students' successful implementation of the "induced function" idea along with various geometric commands of the software (e.g., points, intersection points, line between two points, perpendicular line, tangent line, etc.).

Students appeared to have frequently implemented MRC2, that is, critique and compare the adequacy of representations and judge their suitability for various tasks, in many occasions: (i) Upon realizing that the syntax Limit[f,0] failed, Macy and Kyra proposed graphing the numerator and the denominator functions separately, as a more suitable representation; (ii) Students' questioning of the mathematical fidelity (Dick, 2008) of the software (e.g., not taking for granted everything they see on the screen): (a) When Kyra's Limit[g,1] syntax and the value $b=0$ appeared on the algebra sidebar (Fig. 10a), she questioned the mathematical fidelity as she explained, "it gave me zero but it shouldn't be zero according to the graph it's negative [zooming into the graph (Fig. 10b)]"; (b) Glen's realization: “Maybe graphically you can't really get the exact value but you get a pretty close approximation (Fig. 9c)"; (iii) Gilda's willingness to revise her demonstration for the purpose of illustrating the 
l'Hospital's Rule graphically (in a reconciliatory manner) for the $f(x)=\ln x$ and $g(x)=\cot \frac{\pi x}{2}$ problem; (iv) Students' successful change of strategies to conform the visualization of the l'Hospital's Rule in 2-step problems (Sharon, Gilda, Kyra).

As for MRC3, that is, Understand the purposes of representations generally and in particular contexts and understand how representations do the work they do for us, students offered the following instantiations: (i) Andy's willingness to "graph the derivative of the top divided by the derivative of the bottom and see that as $\mathrm{x}$ goes to zero it should be the same as the original graph" in the investigation of a limit problem of the $0 / 0$ form in an attempt to make sense of the l'Hospital's Rule and reconcile l'Hospital's algorithm with the graphical representation; (ii) Visualization of the $y$-intercept of the original function as the limiting value, in the particular context where the sought limit is at $x=0$ (Gilda, Andy, Kyra, Macy); (iii) Gilda and Sharon's abilities to convert the $0 \cdot \infty$ form to 0 / 0 form in the graph for the purpose of visualizing the l'Hospital's Rule; (iv) Gilda's realization of what makes the 0 / 0 ratio undefined visually: "because it's a horizontal line so regardless of where it is on the graph, because it's a horizontal line that's what makes the ratio undefined."

Among all the MRCs, students seem to have implemented MRC4 the most, that is, Explain representations (i.e., the ability to articulate their competence with the preceding items): (i) Proposing the intersection of the numerator and the denominator function graphs as the point for which the derivative to be evaluated, founded in the argument that "derivative over derivative intersects the same point as the original function intersects at" (Andy, Gilda); (ii) Reference to tangent line as an approximation of secant lines (Glen); (iii) Proposing the undefinedness of the $\tan \left(\mathrm{pi}^{*} x / 2\right)$ function at $x=1$ as a reason for the function $\mathrm{f}$ having a limit at $x=1$ (Sharon); (iv) Confirmation of the undefined-ness of the limit expressions using various techniques with the software (all students); (v) Gilda's inclusion of her "intersection strategy" within the successive tangential visualization: "where the tangent lines intersect that will give you the point... so I want them to intersect at 1 [drags point A towards $x=1$ (Fig. 12c)] because that's the question... I want to know what the limit is at that point;" (vi) In a multi-step problem involving repeated use of the l'Hospital's Rule, Kyra's realization of the similarity (in behaviors) of the functions at each stage near the point of discontinuity [Kyra used this discovery of hers to note that the l'Hospital's Rule could be visualized by noting that all the quotient functions at each stage would behave similarly near the same point of discontinuity]; (vii) Sharon and Gilda's reference to second derivatives of $f$ and $g$ (the numerator and the denominator functions) as another way of visualizing the sought limit value; (viii) Gilda's proposition of a generalized algorithm (recipe) for multi-step l'Hospital's problems in general with reference to the slopes of the tangent lines of the numerator and denominator functions, at each step.

In the context of MRC5, that is, learning new representations quickly and with minimal instruction (diSessa, 2004, p. 293), students appeared to have very strong positive feelings and productive disposition towards the visualization of the l'Hospital's Rule in the dynamic geometry software. In particular, students implemented MRC5 in the following instances: (i) Zooming into the graph technique; awareness of local linearity (Anna, Glen, Gilda ${ }^{6}$ ); (ii) Measuring slope for further confirming the derivative at a point (Glen, Kyra); (iii) Sharon's willingness to drag both points on each function (the numerator and the denominator functions) at the same time triggered by her productive disposition resulting in simultaneous tangential visualization; (iv) Sharon and Gilda's 
discoveries of the second derivative syntax and their implementation of this syntax in connection with the values of the first derivatives ( $f^{\prime}$ and $g^{\prime}$ ); (v) Students' realization of the distinction between two aspects of a line (functional vs. geometric) via the Slope Tool, which worked for the latter case only (Sharon, Gilda); (vi) Sharon's ability to provide multiple visualizations (meta-representational flexibility) within her simultaneous tangential visualization by readjusting the location of the draggable point (being on the quotient $\mathrm{f} / \mathrm{g}$ function plot $v s$. being on the quotient $f^{\prime} / g^{\prime}$ function plot).

\section{Reconciling Multiple Visualizations: Meta- Representational Fluency}

As demonstrated in the analysis chapter, math majors were equipped with the necessary metarepresentational competences in revealing the foundations of the l'Hospital's formalism in a multirepresentational (Duval, 1993) dynamic geometry environment. The results presented above are partly in agreement with Norman and Prichard (1994) who reported that some of their Calculus I students "had difficulty seem reconciling the definition of a derivative of a function at a point with the slope of the tangent line at the point" (p. 75). Out of eight math majors who participated in this study, Glen, Kyra, Sharon, and Gilda have been observed to consistently refer to the notion of "slope of a tangent line" in their visualizations of the derivative functions of the numerator and the denominator functions at a point. These four students' graphical approaches seemed to agree with l'Hospital's (1696) original representation when the problem was of (or convertible to) the 0/0 form. Among these four, Gilda and Sharon consistently referred to tangent lines in all limit problems in their visualizations of the l'Hospital's Rule.

In the exploration of the problems $\lim _{x \rightarrow 1^{+}}\left(\frac{x}{x-1}-\frac{1}{\ln x}\right)$ and $\lim _{x \rightarrow 0^{+}}\left(\frac{1}{x}-\frac{1}{e^{x}-1}\right)$ of the $\infty-\infty$ form, whereas the other students sufficed with the idea of plotting a draggable point on the induced function graph and extracting information by reading the $y$-coordinate of the draggable point; Gilda and Sharon exhibited a variety of mathematical visualization skills in their demonstration of the l'Hospital's Rule. As Zimmermann and Cunningham (1991) put it, mathematical visualization "gives depth and meaning to understanding, serves as a reliable guide to problem solving, and inspires creative discoveries" (p. 4). The participants of the present report not only came up with a diversity of ways that the l'Hospital's Rule could be visualized, but they consistently thought deeply about and made sense of their graphical demonstrations in connection with their analytic derivations. These findings concur with Zimmemann and Cunningham (1991) who postulated, "computer-based visualization, whether static, dynamic, or interactive, is only one facet of the role of computers in mathematics. Visualization must be linked to the numerical and symbolic aspects of mathematics to achieve the greatest results" (p. 5). The findings of the present report - those pertaining to Sharon and Gilda, in particular - therefore, postulates meta-representational fluency as an important aspect in the learning and representing of the l'Hospital's formalism in a dynamic environment.

\section{Implications}

Multiple representations of the l'Hospital's formalism, when integrated with the computing technology, proved as powerful instruments in helping students build solid conceptual foundations in a manner that made sense to them. The participants of the present report achieved a stage of "personally understandable" (Frid, 1994, p. 93) learning of the l'Hospital's Rule, a behavior that was also exhibited by a group of Calculus students, which Frid (1994) named as "connectors." The 
above results are in agreement with the findings of Frid (1994), who observed that connectors "displayed more competence, confidence, and satisfaction in their abilities to do calculus" (p. 93). The significance of multiple representations (Duval, 1993), in particular, multi-representational fluency in calculus is also emphasized by Zimmermann and Cunningham (1991), who postulated that mathematical visualization "gives depth and meaning to understanding, serves as a reliable guide to problem solving, and inspires creative discoveries... Visual thinking and graphical representations must be linked to other modes of mathematical thinking and other forms of representation" (p. 4). Multirepresentational fluency, in the context of the l'Hospital's Rule in particular, can lead students toward strong conceptual development along with a strong sense of connections among the multiple visualizations associated with the limit problem under consideration.

The present report also brings forward the diversity of student-generated ways (global and functional tangential visualizations) that the l'Hospital's Rule can be visualized in a dynamic environment. The dynamic geometry technology and the mathematical freedom provided to students appeared to have influential roles in helping students develop this diversity of visualizations along with deep insight and intuition. As Roberts (1996) stressed: "The role technology can play in helping students construct knowledge and build conceptual understanding is perhaps more important than its role as a tool of calculation and manipulation" (p. 2). The analysis above demonstrated that students not only made a strong sense of the l'Hospital's Rule in a dynamic environment, but they were also "able to give a clear explanation of why things work the way they do, what they mean, when they are to be used" (p. 4).

\section{CONCLUSION}

The present qualitative study on mathematics majors' visualization of the l'Hospital's Rule in a dynamic geometry environment highlighted student-generated representations in a theoretical framework drawn from visual thinking in calculus (Hughes-Hallett, 1991; Zimmermann, 1991) and meta-representational competences (diSessa, 2004) perspectives. Such an approach seemed to provide the research participants with mathematical freedom and productive use of the dynamic geometry technology, which in turn resulted in an awareness of the multiple ways that the l'Hospital's Rule can be visualized in a manner that widened students' repertoire of visual images. Students' expression of meta-representational fluency occurred in different, yet not necessarily hierarchical modes of visualizations. Whereas global tangential visualization appeared in the form of static, successive, or simultaneous visualizations of the tangency points on both function graphs; functional visualization (local-tangential functional, static derivative functional, dynamic derivative functional) appeared as an additional key construct in students' treatment of the l'Hospital's Rule by primarily focusing on the function graphs, without taking tangent lines into account.

Students delivered a snapshot-based approach in a manner that enabled them to develop a span of meta-representational competences in coordination with the geometric constructions that took place. Moreover, whereas tangential visualizations of various types manifested in the case of problems of the forms $0 / 0$ and $\infty / \infty$, such visualizations proved either unavailable or challenging in the case of $0 \cdot \infty$ form. In multi-step problems requiring multiple use of the l'Hospital's Rule, an immediate visualization of the l'Hospital's Rule was not available; students felt the need to borrow from their analytic approach prior to visualizing the l'Hospital's formalism in such situations. Possible extensions to this present study 
could be to investigate students' visualizations of the l'Hospital's Rule in multi-step problems requiring multiple use of the l'Hospital's algorithm in a more structured manner. Further research studies could focus on students' visualization of the l'Hospital's Rule and the emerging meta-representational competences in limit problems involving the three indeterminate forms of exponential type $\left(1^{\infty}, 0^{0}, \infty^{0}\right)$ in a multi-representational dynamic geometry environment. 


\section{APPENDIX A}

Figure Al.

Anna's written work

$$
\begin{array}{r}
\lim _{x \rightarrow 0}\left(\frac{1}{x}\right) \cdot \sin (\pi x) \\
\frac{1}{x} \cdot \sin (\pi x) \\
\frac{\sin (\pi x)}{x} \\
\frac{\cos (\pi x)}{1}=\pi \cos (\pi x) \\
\pi \cos (\pi(0)) \\
\pi \cos (0)=\pi
\end{array}
$$

Figure A3.

Gilda's written work

$$
\begin{aligned}
\lim _{x \rightarrow 0} \frac{8^{x}-2^{x}}{6^{x}-3^{x}}= & \lim _{x \rightarrow 0} \frac{8^{0}-2^{0}}{6^{0}-3^{0}}=\frac{1-1}{1-1}=\frac{0}{0} \\
& \begin{aligned}
8^{x} \ln 8-2^{x} \ln 2 \\
6^{x} \ln 6-3^{x} \ln 3
\end{aligned}=\frac{\frac{\ln 8-\ln 2}{\ln 6-\ln 3}=\frac{\ln 4}{\ln 2}=\frac{2 x^{4}}{x^{2}}}{=2}
\end{aligned}
$$

Figure A4.

Lindsey's written work

$$
\begin{aligned}
& \lim _{x \rightarrow 0} \frac{8^{x}-2^{x}}{6^{x}-3^{x}} \Rightarrow \frac{0}{0} \text { waret. } \\
& =\frac{8^{x}}{6^{x}-3^{x}}-\frac{2^{x}}{6^{x}-3^{x}} \\
& \Rightarrow \frac{(\ln 8) 8^{x}-(\ln 2) 2^{x}}{(\ln 6) 6^{x}-(\ln 3) 3^{x}}=\frac{(\ln 8) 8^{x}}{(\ln 6) 6^{x}-(\ln 3) 3^{x}}-\frac{(\ln 2) 2^{x}}{(\ln 6) 6^{x}-(\ln 3) 3^{x}} \\
& \frac{\ln 8}{\ln 6-\ln 3}-\frac{\ln 2}{\ln 6-\ln 3} \\
& =\frac{\ln 8}{\ln 2}-\frac{\ln 2}{\ln 2}=\frac{\ln (4)}{\ln 2}=2
\end{aligned}
$$


META-REPRESENTATIONAL FLUENCY

Gunhan Caglayan

Figure A5.

Macy's written work

$$
\lim _{x \rightarrow 0} \frac{8^{x}-2^{x}}{6^{x}-3^{x}} \frac{8^{0}-2^{0}}{6^{0} \cdot 3^{\circ}} \frac{\ln 8\left(8^{x}\right)-\operatorname{cn} 2\left(2^{x}\right)}{\ln 6\left(6^{x}\right)-\operatorname{Ln} 3\left(3^{x}\right)} \frac{\operatorname{Lr} 8-\operatorname{Ln}^{2}}{\operatorname{Ln} 6-\operatorname{Ln} 3}
$$

Figure A6

Sharon's written work

$$
\begin{aligned}
& f(x)=\frac{8^{x}-2^{x}}{6^{x}-3^{x}} \quad f(0)=\frac{8^{\circ}-2^{0}}{6^{\circ}-3^{0}} \\
& g^{\prime}(x)=\operatorname{Ln}(8) 8^{x}-\operatorname{Ln}(2) 2^{x} \Rightarrow g^{\prime}(0)=\operatorname{Ln}(8)-\operatorname{Ln}(2) \\
& h^{\prime}(x)=\operatorname{Ln}(6) 6^{x}-\operatorname{Ln}(3) 3^{x} \Rightarrow h^{\prime}(0)=\operatorname{Ln}(6)-\operatorname{Ln}(3)
\end{aligned}
$$

Figure A7.

Kyra's written work

$$
\begin{aligned}
& \lim _{x \rightarrow 1^{+}} \ln x \tan \left(\frac{\pi x}{2}\right)=\lim _{t \rightarrow i^{+}} \frac{\ln x}{\cot \left(\frac{\pi x}{2}\right)} \\
& L \text { Hospital }=\frac{\frac{1}{x}}{\frac{-\frac{\pi}{2} \csc ^{2}}{2}\left(\frac{\pi x}{2}\right)=\frac{1}{-\frac{\pi}{2}}}
\end{aligned}
$$

Figure A8.

Sharon's written work

$$
\begin{aligned}
& \lim _{x \rightarrow 1} h(x)=-\frac{2}{\pi} \\
& \lim _{x \rightarrow 1} \frac{f(x)}{g(x)}=\lim _{x \rightarrow 1} \frac{f^{\prime}(x)}{g^{\prime}(x)}=\frac{1.00}{-1.5708}
\end{aligned}
$$

93 
Figure A9.

Gilda's first attempt

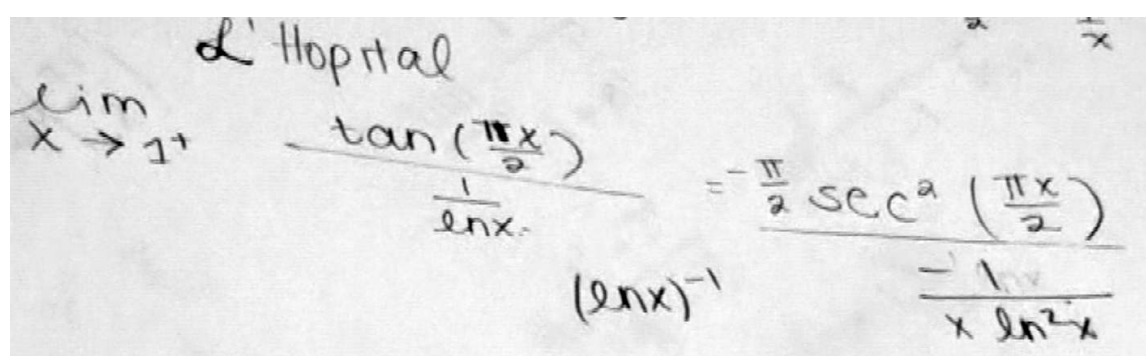

Figure A10.

Gilda's change of mind

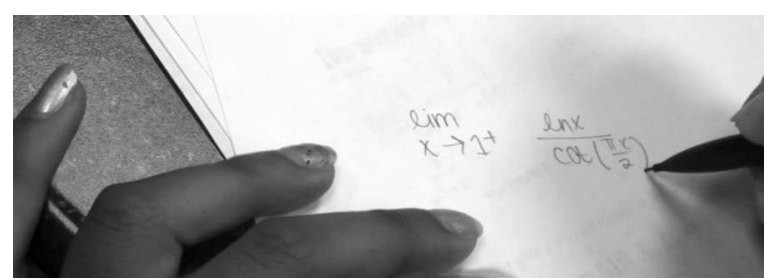

Figure A11.

Gilda's complete solution after her change of mind

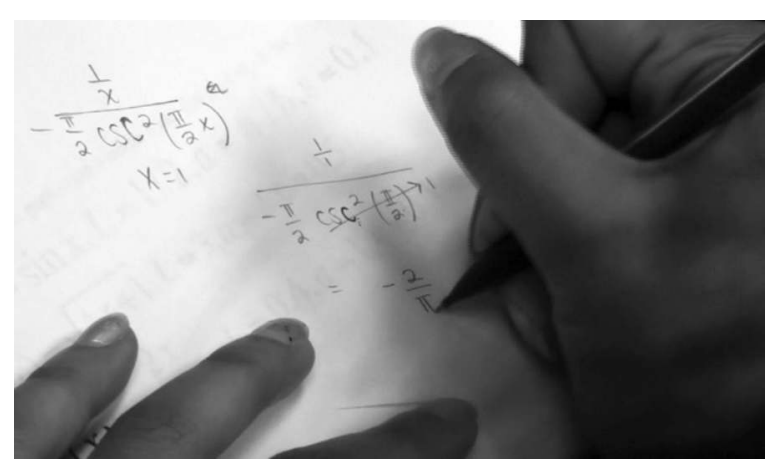

Figure A12.

Kyra's written work

$$
\begin{aligned}
& \lim _{x \rightarrow 1^{+}} \frac{x-(x-1)}{(x+1) \ln x} \quad=\lim _{i=} \frac{x \ln x-1}{x \ln x-\ln x} \\
& \frac{d\left[\frac{\ln y-x-1]}{d e}\right.}{\frac{d[+\ln -\ln x]}{d x}}=\frac{1 m+1-1}{\ln x+1-\frac{1}{x}}=\frac{1-x}{\ln x+1-1 / x}=\frac{\ln (1)}{\ln (x)+1-(1)} \\
& \frac{d[1, x] / d x}{\frac{d[\ln x+1-1 / x]}{d x}}=\frac{\frac{1}{x}}{\frac{d x+1 / x^{2}}{x}}=\frac{x^{-1}}{x^{-1}+x^{-2}} \cdot \frac{x^{-1}}{x^{-1}\left(1+x^{-1}\right)}-\frac{1}{1+x^{-1}}=\frac{1}{2}
\end{aligned}
$$


Figure A13.

Gilda's written work

$$
\begin{aligned}
& \lim _{x \rightarrow 1^{+}}\left(\frac{x}{x-1}-\frac{1}{\ln x}\right)^{1} \frac{x \ln x-x+1}{\ln x(x-1)} \quad \frac{1 \ln 1^{70}-1+1}{\ln 1(1-1)}=\frac{0}{0} \\
& \text { 1. Hoprial } \\
& \lim _{x \rightarrow 1^{+}} \frac{x \ln x-x+1}{(x-1) \ln x}=\lim _{x \rightarrow 1^{+}} \frac{x \cdot \frac{1}{x}+\ln x-1}{(x-1) \frac{1}{x}+\ln x}=\lim _{x \rightarrow 1^{+}} \frac{1-1^{0}+\ln x}{1-\frac{1}{x}+\ln x} \\
& \uparrow \\
& \begin{array}{l}
\lim _{x \rightarrow 1^{+}} \frac{\ln x}{1-\frac{1}{x}+\ln x} \quad x=\frac{0}{0} \\
\lim _{x \rightarrow 1^{+}} \frac{\frac{x+x^{2}}{x^{3}}}{x^{2}}=\frac{1}{x}\left(\frac{x^{2}}{x+x^{2}}\right)
\end{array} \\
& \lim _{x \rightarrow 1^{+}} \frac{x^{2}}{x+x^{2}}=\frac{1}{2}
\end{aligned}
$$

\section{APPENDIX B}

Snapshot 1 .

Static tangential visualization

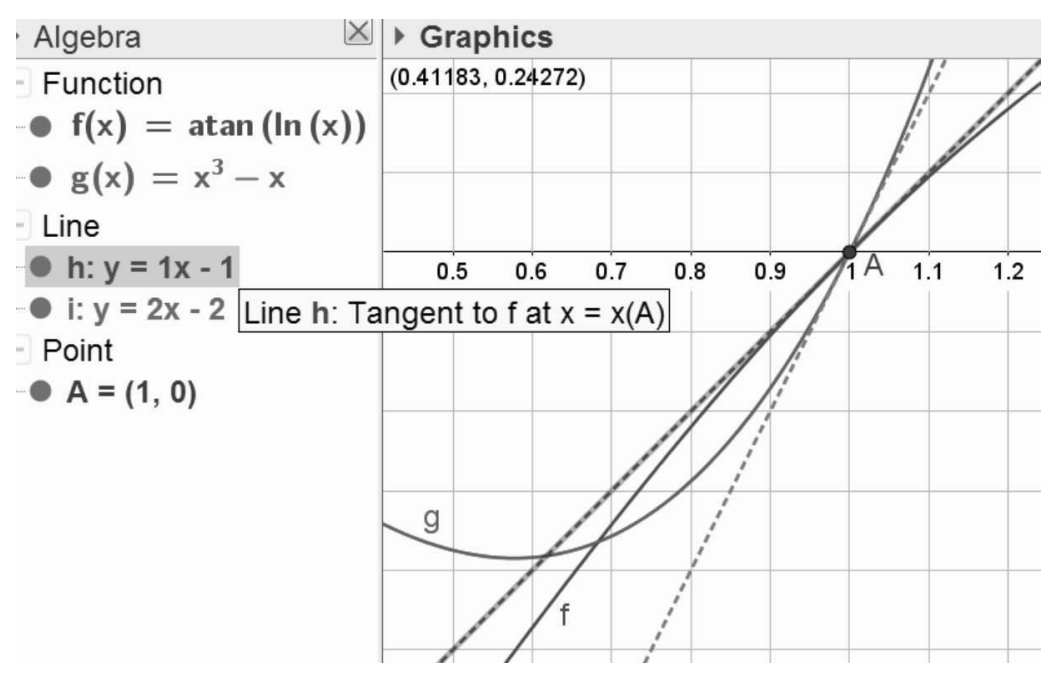


Snapshot $2 a$.

Successive (consequential) tangential visualization (Pseudo-Dynamic)

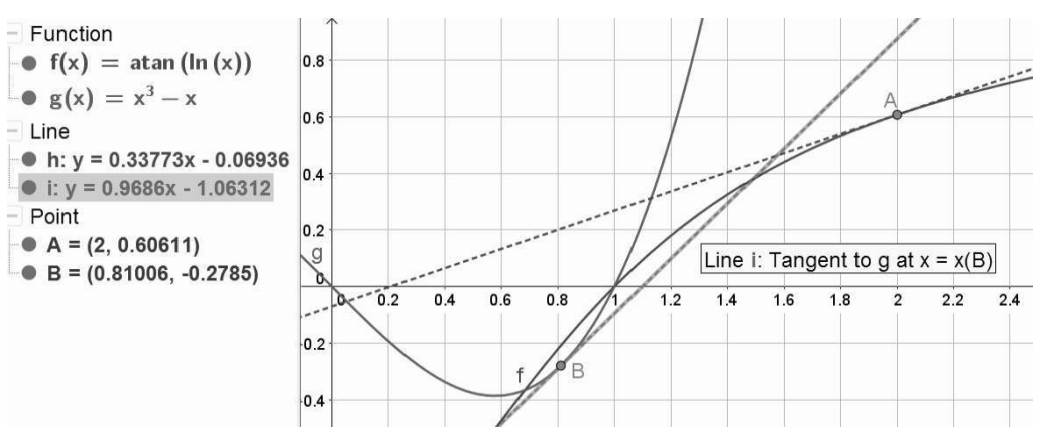

Snapshot $2 b$.

Points A and B are dragged until they meet at the common point of tangency $(1,0)$

\section{Function}

- $\mathrm{f}(\mathrm{x})=\operatorname{atan}(\ln (\mathrm{x}))$

$\mathrm{g}(\mathrm{x})=\mathrm{x}^{3}-\mathrm{x}$

Line

- $\mathrm{h}: \mathrm{y}=\mathrm{x}-1$

i: $y=1.99023 x-1.99024$

Point

$A=(1,0)$

$B=(0.99837,-0.00325)$

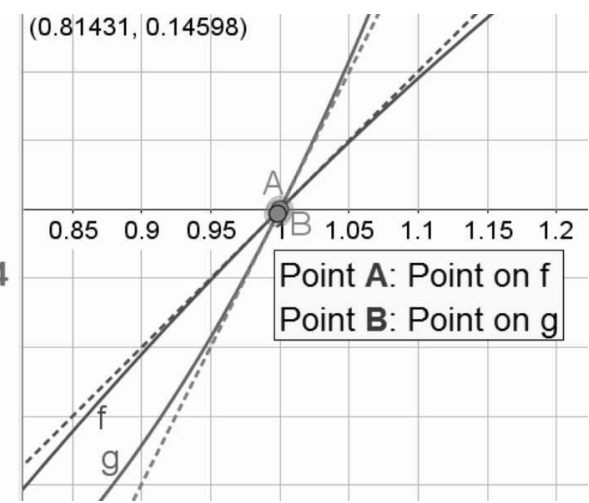

Snapshot 3a.

Simultaneous (coherent) tangential visualization (Dynamic)
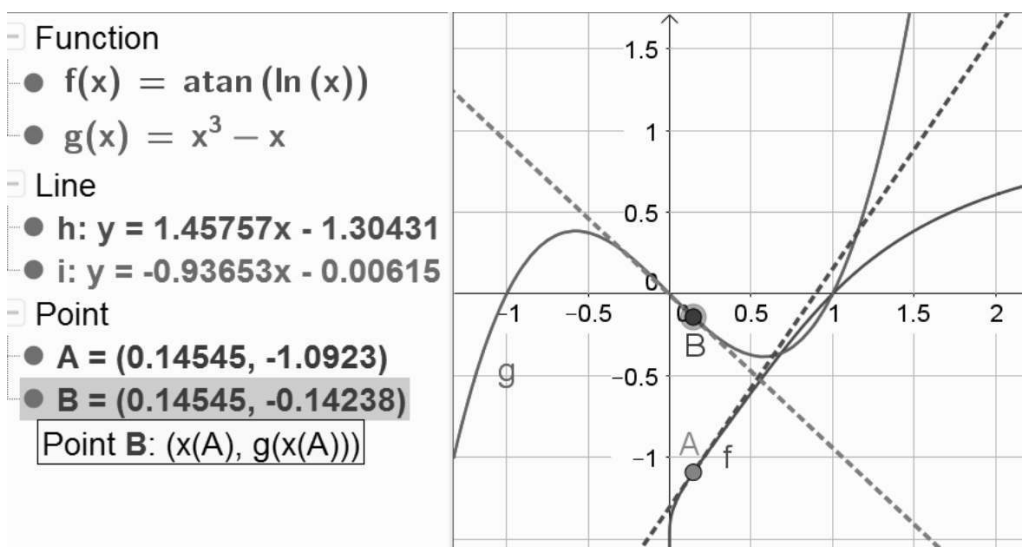
Snapshot 3b.

Point A is dragged until Points A and B meet at the common point of tangency $(1,0)$

Function
$\mathrm{f}(\mathrm{x})=\operatorname{atan}(\ln (\mathrm{x}))$
$\mathrm{g}(\mathrm{x})=\mathrm{x}^{3}-\mathrm{x}$
$\mathrm{Line}$
$\mathrm{h}: \mathrm{y}=\mathrm{x}-1$
i: $\mathrm{y}=2 \mathrm{x}-2$
Point
$\mathrm{A}=(1,0)$
$\mathrm{B}=(1,0)$

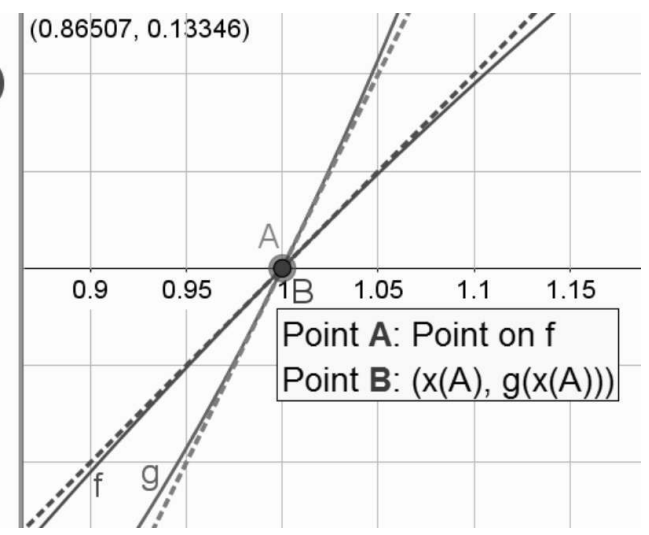

Snapshot 4.

Local-Tangential (Functional) Visualization

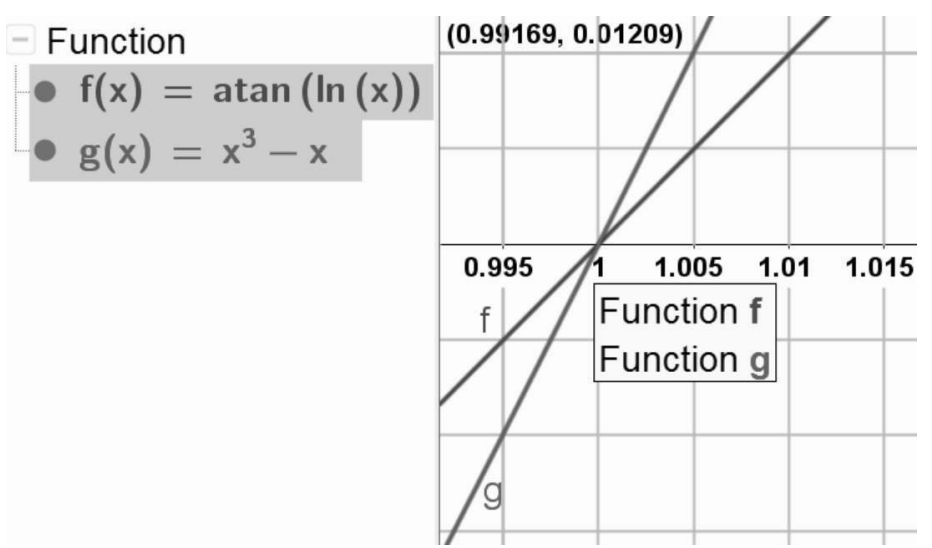

Snapshot 5.

Derivative Functional Visualization (Static)

$$
\begin{aligned}
& \text { Function } \\
& f(x)=\operatorname{atan}(\ln (x)) \\
& g(x)=x^{3}-x \\
& p(x)=\frac{1}{x x^{2}-1} \\
& \text { Function } p: p(x)=f^{\prime}(x) / g^{\prime}(x) \\
& A=(1,0) \\
& B=(1,0) \\
& C=(1,0.5)
\end{aligned}
$$

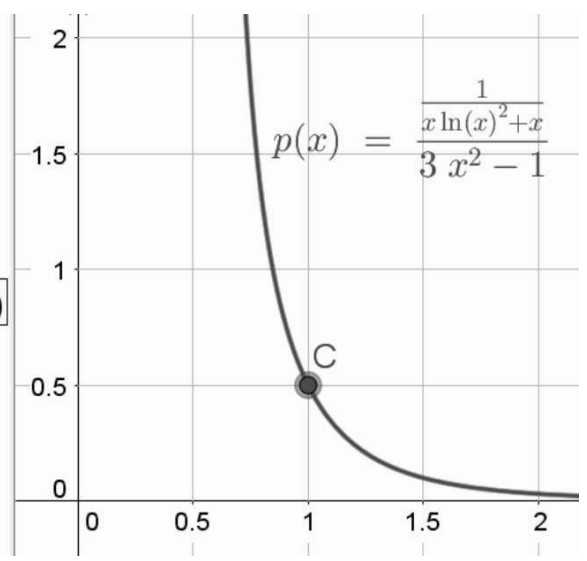


Snapshot 6a.

Derivative Functional Visualization (Dynamic)

- Function

$f(x)=\operatorname{atan}(\ln (x))$

$f^{\prime}(x)=\frac{1}{x \ln (x)^{2}+x}$

$g(x)=x^{3}-x$

- $g^{\prime}(x)=3 x^{2}-1$

Point

$A=(0.45559,1.35654)$

$B=(0.45559,-0.37731)$

Point B: $\left(x(A), g^{\prime}(x(A))\right)$

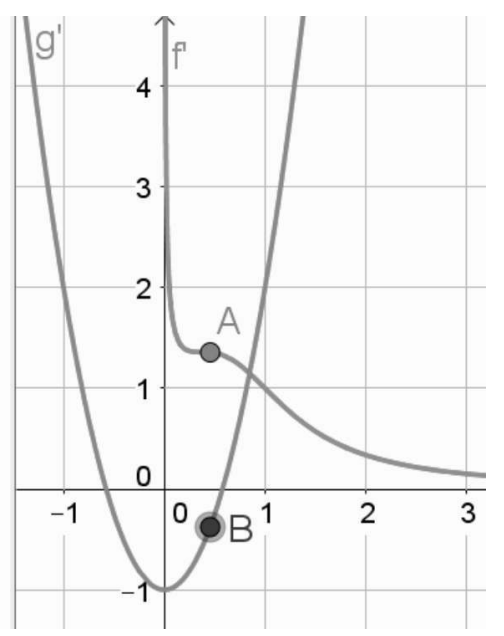

Snapshot 6b.

Point A is dragged until Points A and B both have an abscissa value of $x=1$

$$
\begin{aligned}
& \text { Function } \\
& \mathrm{f}^{\prime}(\mathrm{x})=\frac{1}{\mathrm{x} \ln (\mathrm{x}))^{2}+\mathrm{x}} \\
& \mathrm{g}(\mathrm{x})=\mathrm{x}^{3}-\mathrm{x} \\
& \mathrm{g}^{\prime}(\mathrm{x})=3 \mathrm{x}^{2}-1
\end{aligned}
$$

- Point

$A=(1,1)$

$B=(1,2)$

Point B: $\left(x(A), g^{\prime}(x(A))\right)$

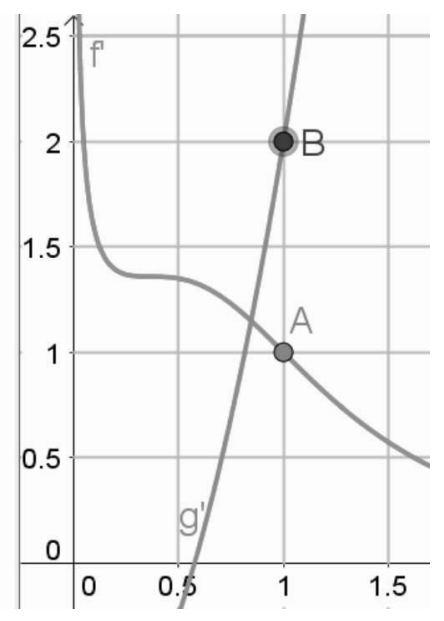

Snapshot 6c.

The additional step of evaluating the ratio of ordinates $y(A) / y(B)$

\section{Function}

$\mathrm{f}(\mathrm{x})=\operatorname{atan}(\ln (\mathrm{x}))$

$f^{\prime}(x)=\frac{1}{x \ln (x)^{2}+x}$

$g(x)=x^{3}-x$

$\mathrm{g}^{\prime}(\mathrm{x})=3 \mathrm{x}^{2}-1$

Number

$$
a=1 / 2
$$

Number a: $y(A) / y(B)$

- $A=(1,1)$

- $B=(1,2)$

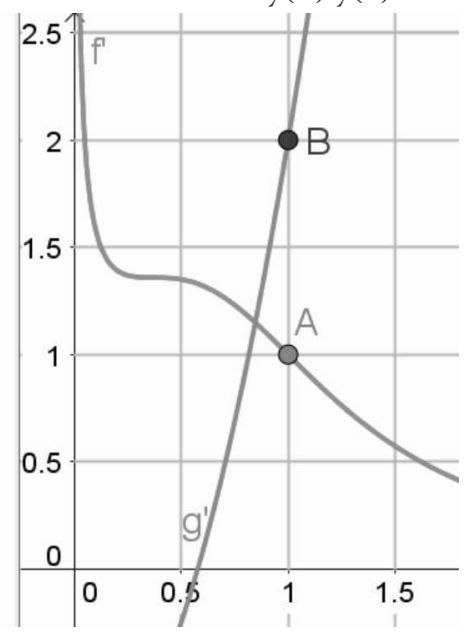


Gunhan Caglayan

\section{NOTES}

1. All snapshots are presented in Appendix B.

2. The asterix "*” in GeoGebra is used to denote multiplication.

3. Students' written works are presented in Appendix A.

4. The phrase "induced function" refers to the function equation students came up with in order to find the limit of the expression under consideration.

5. Because the software graphs any expression in $x$ typed in the input bar by assigning a function equation, I used the phrase "induced function" for all such occurrences in which students embraced this approach.

6. Gilda's discovery of the local-linearity is made very explicit in her usage: "I use the derivative function graphs as my tangent lines so I was able to find the limit without actually making the tangent lines."

\section{RÉFÉRENCES}

Amit, M., \& Vinner, S. (1990). Some misconceptions in calculus: Anecdotes or the tip of the iceberg? In G. Booker, P. Cobb, \& T. N. de Mendicuti (Eds.), Proceedings of the 14th International Conference for the Psychology of Mathematics Education, Vol. 1 (pp. 3-10). Oaxtepec, Mexico: CINVESTAV.

Artigue, M. (1991). Analysis. In D. Tall (Ed.), Advanced mathematical thinking (pp. 167-198). Dordrecht: Kluwer Academic Press.

Asiala, M., Cottrill, J., Dubinsky, E., \& Schwingendorf, K. E. (1997). The development of students' graphical understanding of the derivative. Journal of Mathematical Behavior, 16(4), 399-431.

Bernard, H. (1994). Research methods in anthropology (2nd ed.). Thousand Oaks, CA: Sage.

Bingolbali, E., Monaghan, J., \& Roper, T. (2007). Engineering students' conceptions of the derivative and some implications for their mathematical education. International Journal of Mathematical Education in Science and Technology, 38(6), 763-777.

Bingolbali, E., \& Monaghan, J. (2008). Concept image revisited. Educational Studies in Mathematics, 68(1), 19-35.

Biza, I., Christou, C., \& Zachariades, T. (2008). Student perspectives on the relationship between a curve and its tangent in the transition from Euclidean Geometry to Analysis. Research in Mathematics Education, 10(1), 53-70.

Biza, I., \& Zachariades, T. (2010). First year mathematics undergraduates' settled images of tangent line. Journal of Mathematical Behavior, 29, 218-229.
Borwein, J. M., \& Bailey, D. H. (2003). Mathematics by experiment: Plausible reasoning in the 21 st century. AK Peters Ltd.

Borwein, J. M. (2005). The experimental mathematician: The pleasure of discovery and the role of proof. International Journal of Computers for Mathematical Learning, 10, 75-108.

Boyatzis, R. (1998). Transforming qualitative information: Thematic analysis and code development. Thousand Oaks, CA: Sage Publications.

Bremigan, E. (2005). Analysis of diagram modification and construction in students' solutions to applied calculus problems. Journal for Research in Mathematics Education, 36, 248-277.

Castela, C. (1995). Apprendre avec et contre ses connaissances antérieures: Un example concret, celui de la tangente. Recherches en Didactiques des Mathématiques, 15(1), 7-47.

Cornu, B. (1991). Limits. In D. Tall (Ed.), Advanced mathematical thinking (pp. 153-166). Dordrecht: Kluwer.

Davis, R. B., \& Vinner, S. (1986). The notion of limit: Some seemingly unavoidable misconception stages. Journal of Mathematical Behavior, 5(3), 281-303.

Dick, T. P. (2008). Keeping the faith: Fidelity in technological tools for mathematics education. In G. W. Blume $\&$ M. K. Heid (Eds.), Research on technology and the teaching and learning of mathematics: Vol. 2. Cases and perspectives (pp. 333-339). Charlotte, NC: Information Age.

Dick, T. P., \& Edwards, B. S. (2008). Multiple representations and local linearity: Research influences on the use of technology in calculus curriculum reform. In G. W. Blume \& M. K. Heid (Eds.), Research on technology and the teaching and learning of mathematics: Vol. 2. Cases and perspectives (pp. 255-275). Charlotte, NC: Information Age.

diSessa, A. A. (1988). Knowledge in pieces. In G. Forman $\&$ P. Pufall (Eds.), Constuctivism in the computer age (pp. 49-70). Hillsdale, NJ: Lawrence Erlbaum Associates.

diSessa, A. A. (2004). Metarepresentation: Native competence and targets for instruction. Cognition and Instruction, 22(3), 293-331.

Duval, R. (1993). Registres de représentation sémiotique et fonctionnement cognitif de la pensée. Annales de Didactique et de Sciences Cognitives, 5, 37-65.

Eves, H. (2006). An introduction to the history of mathematics ( $6^{\text {th }}$ edition). Brooks/Cole - Thomson Learning.

Ferrini-Mundy, J., \& Graham, K. (1994). Research in calculus learning: Understanding of limits, derivatives, and integrals. In J. J. Kaput \& E. Dubinsky (Eds.), Research issues in undergraduate mathematics learning, MAA Notes (Vol. 33, pp. 31-46). Washington, D. C: Mathematical Association of America.

Frid, S. (1994). Three approaches to calculus instruction: Their nature and potential impact on students' language use and sources of conviction. In E. Dubinsky, A. Schoenfeld \& J. Kaput (Eds.), Research in Collegiate 
Mathematics Education I (pp. 69-100). Washington, D. C: Conference Board of the Mathematical Sciences.

Gass, F. (1992). Limits via graphing technology. PRIMUS, 2(1), 9-15.

Glaser, B. G., \& Strauss, A. L. (1967). The discovery of grounded theory: Strategies for qualitative research. Chicago: Aldine.

González-Martín, A., Bloch, I., Durand-Guerrier, V., Maschietto, M. (2014). Didactic Situations and Didactical Engineering in University mathematics: cases from the study of Calculus and proof. Research in Mathematics Education, 16(2) 117-134.

Harrington, W. J. (2006). Student understanding of the concept of limit in a technological environment. In L. R. van Zoest (Ed.) Teachers engaged in research: inquiry into mathematics classrooms, grades 9-12 (pp.39-57). Greenwich, CT: IAP-Information Age Publications.

Hughes-Hallett, D. (1991). Visualization and calculus reform. In W. Zimmermann \& S. Cunningham (Eds.), Visualization in teaching and learning mathematics (pp. 121-126). Washington, DC: Mathematical Association of America.

Izsak, A., Caglayan, G., \& Olive, J. (2009). Metarepresentation in a Algebra I classroom. Journal of the Learning Sciences, 4, 549-587.

Katz, V. J. (2009). A history of mathematics, an introduction, third edition. Pearson.

Kleiner, I. (2001). History of the infinitely small and the infinitely large in calculus. Educational Studies in Mathematics, 48, 137-174.

Kvale, S. (2007). Doing interviews. London: Sage.

Lecorre, T. (2015). Définir: une nécessité à construire. Le cas de la définition de la limite d'une fonction. Repères Irem, 100, 51-64.

Lesh, R., Post, T., \& Behr, M. (1987). Representations and translations among representations in mathematics learning and problem solving. In C. Janvier, (Ed.), Problems of representations in the teaching and learning of mathematics (pp. 33-40). Hillsdale, NJ: Lawrence Erlbaum.

Mamolo, A., \& Zazkis, R. (2012). Stuck on convention: A story of derivative-relationship. Educational Studies in Mathematics, 81(2), 161-167.

Mamona-Downs, J. (2001). Letting the intuitive bear on the formal: a didactical approach for the understanding of the limit of a sequence. Educational Studies in Mathematics, 28, 259-288.

National Council of Teachers of Mathematics (2000). Principles and standards for school mathematics. Reston, VA: Author

Norman, F. A., \& Prichard, M. K. (1994). Cognitive obstacles to the learning of calculus: a Krutetskiian perspective. In J. Kaput \& E. Dubinsky (Eds.), Research issues in undergraduate mathematics learning: Preliminary analyses and results (pp. 63-78). Mathematical Association of America.
Oehrtman, M. (2009). Collapsing dimensions, physical limitation, and other student metaphors for limit concepts. Journal for Research in Mathematics Education, 40(4), 396-426.

Orton (1983). Students' understanding of differentiation. Educational Studies in Mathematics, 14(3), pp. 235-250.

Patton, M. Q. (2002). Qualitative research and evaluation methods. Thousand Oaks, CA: Sage.

Przenioslo, M. (2004). Images of the limit of function formed in the course of mathematical studies at the university. Educational Studies in Mathematics, 55, 103-132.

Robert, A. (1982). Lacquisition de la notion de convergences des suites numériques dans l'enseignement supérieur. Recherches en Didactique des Mathématiques, 3(3), 305-341.

Roberts, W. (1996). A modern course in calculus. In W. Roberts (Ed.), Calculus: The dynamics of change (pp. 1-5). Washington, D.C: Mathematical Association of America.

Robinet, J. (1983). Une expérience d'ingénierie didactique sur la notion de limite de fonction. Recherches en Didactique des Mathématiques, 4(3), 232-292.

Rogalski, M. (1990). Comment étudier la convergence d'une suite réelle? Un exemple de méthode. Commission InterIREM Université Enseigner autrement les mathématiques en DEUG A première année (pp. 197-204). Lyon, Paris: IREM Université Paris-Diderot.

Rogalski, M. (2015). De la notion couplée de tangente et dérivée à la notion de limite. In Autour de la notion de limite en classe de première scientifique, Brochure Irem $\mathrm{n}^{\circ} 97$, Irem de l'Université Paris-Diderot.

Rogalski, M. (2016). Revenir au sens de la notion de limite par certaines de ses raisons d'être: un chantier pour le début de l'analyse à l'université. Retrieved from [https:// hal.archives-ouvertes.fr/hal-01337945/].

Schneider, M. (2001). Praxéologies didactiques et praxéologies mathématiques. A propos d'un enseignement des limites au secondaire. Recherches en Didactique des Mathématiques, 21(1.2), 7-56.

Sinclair, N. (2008). Computer-based technologies and plausible reasoning. In M. P. Carlson \& C. Rasmussen (Eds.), Making the connection: Research and practice in undergraduate mathematics (pp. 233-244). Washington, DC: Mathematical Association of America.

Sofronas, K. S., DeFranco, T. C., Vinsonhaler, C., Gorgievski, N., Schroeder, L., \& Hamelin, C. (2011). What does it mean for a student to understand the first-year calculus? Perspectives of 24 experts. The Journal of Mathematical Behavior, 30(2), 131-148.

Stewart, J. (2012). Calculus: Early Transcendentals. Cengage.

Struik, D. J. (1963). The origin of l'Hopital's rule. Mathematics Teacher, 56(4), 257-260.

Swinyard, C. (2011). Reinventing the formal definition of limit: The case of Amy \& Mike. Journal of Mathematical Behavior, 30, 93-114. 
Swinyard, C., \& Larsen, S. (2012). Coming to understand the formal definition of limit: Insights gained from engaging students in reinvention. Journal for Research in Mathematics Education, 43(4), 465-493.

Szydlik, J. E. (2000). Mathematical beliefs and conceptual understanding of the limit of a function. Journal for Research in Mathematics Education, 31(3), 258-276.

Tall, D. (1987). Constructing the concept image of a tangent. In J. C. Bergeron, N. Herscovics, \& C. Kieran (Eds.), Proceedings of the 11th PME international conference (Vol. 3, pp. 69-75). Montréal, Canada.

Tall, D. (1991). The psychology of advanced mathematical thinking. In D. Tall (Ed.), Advanced Mathematical Thinking (pp. 3-23). Kluwer: The Netherlands.

Tall, D., \& Vinner, S. (1981). Concept image and concept definition with particular reference to limits and continuity. Educational Studies in Mathematics, 12, 151-169.

Thomas, M. O. J., \& Holton, D. (2003). Technology as a tool for teaching undergraduate mathematics. In
A. J. Bishop, M. A. Clements, C. Keitel, J. Kilpatrick \& F. K. S. Leung (Eds.), Second international handbook of mathematics education (pp. 347-390). London: Kluwer.

Williams, S. R. (1991). Models of limit held by college calculus students. Journal for Research in Mathematics Education, 22(3), 219-236.

Zimmerman, W. (1991). Visual thinking in calculus. In W. Zimmerman \& S. Cunningham (Eds.), Visualization in teaching and learning mathematics (pp. 127-137). Washington, DC: Mathematical Association of America.

Zimmerman, W., \& Cunningham, S. (1991). Editor's introduction: What is mathematical visualization? In W. Zimmerman \& S. Cunningham (Eds.), Visualization in teaching and learning mathematics (pp. 1-8). Washington, DC: The Mathematical Association of America. 